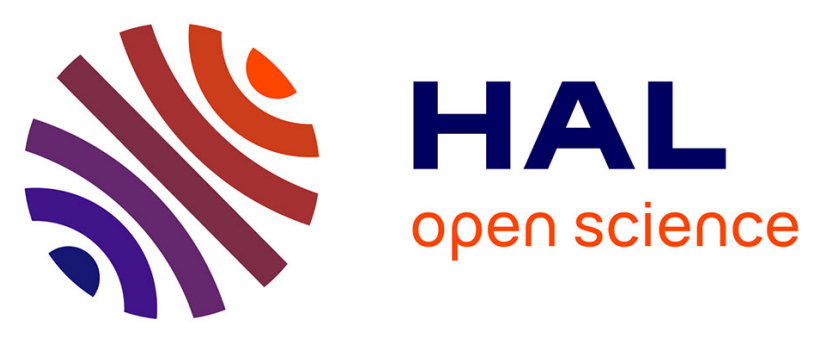

\title{
Investigation of hydrodynamic/biomass growth coupling in a pilot scale granular bioreactor at low pore Reynolds number
}

\author{
Seyed Mohsen Karrabi, Philippe Séchet, Christophe Morra, Alain H. \\ Cartellier, Christian Geindreau, Jean M.F. Martins
}

\section{To cite this version:}

Seyed Mohsen Karrabi, Philippe Séchet, Christophe Morra, Alain H. Cartellier, Christian Geindreau, et al.. Investigation of hydrodynamic/biomass growth coupling in a pilot scale granular bioreactor at low pore Reynolds number. Chemical Engineering Science, 2011, 66 (8), pp.1765-1782. 10.1016/j.ces.2011.01.010 . insu-00648704

\section{HAL Id: insu-00648704 \\ https://hal-insu.archives-ouvertes.fr/insu-00648704}

Submitted on 3 Sep 2020

HAL is a multi-disciplinary open access archive for the deposit and dissemination of scientific research documents, whether they are published or not. The documents may come from teaching and research institutions in France or abroad, or from public or private research centers.
L'archive ouverte pluridisciplinaire HAL, est destinée au dépôt et à la diffusion de documents scientifiques de niveau recherche, publiés ou non, émanant des établissements d'enseignement et de recherche français ou étrangers, des laboratoires publics ou privés. 


\title{
Investigation of hydrodynamic/biomass growth coupling in a pilot scale granular bioreactor at low pore Reynolds number
}

\author{
M. Karrabi ${ }^{a}$, P. Séchet ${ }^{\mathrm{a}, *}$, Ch. Morra ${ }^{\mathrm{a}, \mathrm{b}}$, A. Cartellier ${ }^{\mathrm{a}}$, Ch. Geindreau ${ }^{\mathrm{c}}$, J.M.F. Martins ${ }^{\mathrm{b}}$ \\ ${ }^{a}$ Laboratoire des Ecoulements Géophysiques et Industriels, CNRS/UJF/G-INP, UMR 5519, BP 53 38041, Cedex 09 Grenoble, France \\ ${ }^{\mathrm{b}}$ Laboratoire des Transferts en Hydrologie et Environnement, CNRS/UJF/G-INP/IRD, UMR 5564, BP 53 38041, Cedex 09 Grenoble, France \\ ${ }^{\mathrm{c}}$ Laboratoire Sol-Solide-Structure-Risque, CNRS/UJF/G-INP, UMR 5521, BP 5338041 Cedex 09, Grenoble, France
}

\begin{abstract}
Through a coupled experimental/modelling approach, this study investigates the functioning of a fixed bed bioreactor of Biolite beads, inoculated with Pseudomonas putida, in order to biotreat phenol contaminated waters at low Reynolds number. In particular the coupling between water flow and biomass growth is studied through the evaluation of the relationship between bed permeability reduction and biomass content as well as hydrodynamic effects on biological kinetics. In term of bioclogging, our results showed a very sharp decrease of the relative bed permeability well correlated with measured biofilm growth (for relative permeability greater than 0.8 ) as well as a saturation of the bed permeability at low relative porosity. However, most of the models available in the literature failed to describe our observed results. Our work permitted to show a strongly heterogeneous longitudinal biofilm development along the bed and thus a strongly heterogeneous longitudinal occupation of the porosity that decreased rapidly at the bed entry. Furthermore, the observed measured porosity saturation was shown to correspond to a biomass distribution involving the existence of channelling, through a growth/detachment equilibrium, rather than a complete clogging of the bed reactor. Biofilm microstructure built at the pore scale by bacteria (e.g. via the modulation of EPS production) probably explains the observed permeability/porosity results in the early stage of bed bioclogging. However, the present experiments do not allow measuring such a microstructuration effect. Through the analysis of a simple 1D model written at the mesoscale, we showed that the closure laws accounting for the different biological kinetics (growth, detachment, etc.) are flow dependent, as they represent effective properties and complex biological processes averaged at the mesoscale. Given the low Reynolds number investigated in the present paper, this dependency appears not only controlled by mass transfer evolution but may also involve more complex biological regulation (biological response to physical chemical stresses). As a consequence, formulations that are strictly valid at the cell scale (e.g. Monod formulation) should be used with care if no averaging or upscaling methods are used. The main conclusion of the present work is that the knowledge of macroscopic laws, such as the permeability-porosity relationship is not sufficient to account for the coupling between hydrodynamics and biomass growth in porous media. As bacterial biofilms are formed of populations able to adapt their metabolism to their evolving microenvironment, and in particular to hydrodynamic conditions, it appears that the predictive character of such models could be largely improved if these closure laws were not postulated a priori but deduced either from upscaling techniques, or, as in this paper, from $a d$ hoc experiments performed at the global scale.
\end{abstract}

\section{Introduction}

In the last two decades, a lot of work has been done to optimise the design and the operating conditions of bioreactors for water treatment, from the biofilm scale up to the pilot scale. However, despite this important work, the available models are not yet able to predict correctly the evolution of reactors with

\footnotetext{
* Corresponding author.

E-mail address: philippe.sechet@grenoble-inp.fr (P. Séchet).
}

fixed biomass, both in terms of degradation efficiency and bioclogging.

At the biofilm scale, numerous papers deal with the modelling of biofilm morphology and development on planar or spherical substrata (Wanner et al., 2004). Some of these models, using cellular automata, allow a refined description of the coupling between the flow and the biofilm structure (Picioreanu, 1999; Picioreanu et al., 2000). Recent works deal not only with the hydrodynamics influence on biofilm structure, such as its shape or composition (e.g. Peyton, 1996; Venugopalan et al., 2005; Simoes et al., 2007), but also on cell metabolism itself and the 
effects induced on the structural and kinetic properties of biofilms. At this level, the effect of hydrodynamic conditions on biofilm behaviour arises from many coupled processes and is not yet fully understood (Liu and Tay, 2002; Simoes et al., 2007). For instance, Qi et al. (2008) demonstrated that the EPS production rate is highly dependent on the shear stress and Tsai (2005) reported a water velocity effect on the dynamic behaviour of bacterial biofilm and its development rate. Altogether, these works showed that biofilms are complex communities of cells, able to regulate their metabolism and adapt themselves to various external parameters: heat, $\mathrm{pH}$, substrate availability but also hydrodynamic stress such as shear or pressure forces. No model accounts for these physical effects.

At the porous medium scale, experimental and numerical contributions are available on the coupling between the local hydrodynamics and biofilm growth. Experimental works performed on micro-columns or flowcells allowed reaching conclusions in terms of permeability reduction modelling (Cunningham et al., 1991; Taylor and Jaffé, 1990; Vandevivere and Baveye, 1992; Vandevivere et al., 1995; Vandevivere, 1995; Clement et al., 1996). The reduction level was shown to be controlled by several factors involving pore geometry, biofilm organisation in the porous media (continuous biofilm, aggregates, colonies) as well as the possible variable production of EPS, which depends on the strain and feeding conditions (Vandevivere and Baveye, 1992; Shaw et al., 1985). In particular, this later finding showed that the clogging process of porous media by living material is very different from the clogging by inert material (classical filtration) and that the Kozeny law (or related formula) is not always relevant for the prediction of porous media clogging by bacterial biofilms. Similar works were performed to study the dispersive properties of such media and especially the longitudinal dispersion. Results showed that the evolution of the dispersion coefficient was very difficult to predict. Indeed, this coefficient strongly depends on biomass distribution within the pores and over the bed cross-section. Depending on these distributions and the clogging, the axial dispersion can increase or decrease with the amount of biomass (Bielefeldt et al., 2002). At the pilot scale, there are fewer experiments explicitly dealing with the coupling between hydrodynamics and biomass growth (Deront et al., 1998; Di Iaconi et al., 2006; Menoret, 2001). On the modelling side, several approaches exist. Kildsgaard and Engesgaard (2001) or Brovelli et al. (2009) incorporated directly permeability formulations derived from micro-scale experiments in a macroscopic model. Other approaches consisted in deriving macroscopic laws and their associated effective permeability using upscaling techniques (Wood and Ford, 2007; Anthony et al., 2005; Golfier et al., 2009). Finally, in the frame of fixed biomass reactors modelling, recent available models allow the description of various physical processes occurring at the different scales using network models (Chen-Charpentier, 1999; Thullner et al., 2002) or hierarchical models.

Studying the coupling between hydrodynamic and biofilm growth is a complex task, because of the multiple scales involved as well as the complex behaviour of the biomass (not only in term of spatial organisation but also in term of bacteria cells response to environmental and physico-chemical stress). As a consequence, very few models account for this complexity. The most complete one was proposed by Kapellos et al. (2007), who combined a continuum approach for the description of fluid flow and the solute transport with a cellular automaton approach for the description of biofilm growth at the pore scale. Some features accounting for the biomass metabolism are also included, such as cell growth inhibition under pressure effects or cell-to-cell signalling molecule (Davies et al., 1998).

However, these works leave opened a number of issues, such as the coupling between biomass growth and hydrodynamics that are still not well understood: if some authors (Brovelli et al., 2009) state that the knowledge of the permeability-biomass content is a critical issue, experiments on biofilm itself (Simoes et al., 2007) showed that the hydrodynamic effect on the biofilm may be more complex than initially thought and not only controlled by mechanical effects but also by biological effects. Futhermore, whatever the sophistication of existing models in terms of processes and scales considered, their testing or validation need hardly available data such as global permeability and porosity (Kapellos et al., 2007) or total head loss (Kildsgaard and Engesgaard, 2001). Using such a limited set of variables may not be reliable enough: for instance, different biomass profiles inside a porous media can lead to the same total head loss. There is thus a clear need for coherent databases that can be safely used both for model validation and for the investigation of biofilter dynamics.

To better understand the complex interactions occurring in bioreactors, experiments have been undertaken on a laboratory scale biofilter to investigate the coupling between biomass growth and hydrodynamic at the macroscale as well as its consequences on the pollution removal efficiency. In order to provide a reliable database, care was taken to ensure well controlled experimental conditions and also to perform independent measurements of as many "global" parameters as possible. In the present investigation, the time evolution of the permeability, the porosity and the biomass concentration were independently measured along the biofilter height. So doing, and contrary to what was usually done in most previous experiments (Deront et al., 1998; Menoret, 2001; Di Iaconi et al., 2006), the biofilter dynamics can be investigated without introducing any assumption such as those concerning for example the biofilm structure at the pore scale or the permeability reduction-biomass content relationship.

The paper is organised as follows. In the second section, the experimental setup, the experimental protocols and the measuring techniques are presented. Because of the complex behaviour of bubble flows in packed bed from one side, and biomass growth in porous media on the other side, it was chosen in the present paper not to inject gas but to perform experiment with water presaturated with oxygen. The physical model for bubbly flow in packed bed developed in Bordas et al. (2006) could have been used, but it is, at the present time, difficult to separate the effect of the gas flow from the effect of the biofilm on the pressure drop without a clear knowledge of the involved processes in absence of bubbles. The raw data results are presented and discussed in the third section. In particular, the collected data are first analysed in the frame of the relationship between porosity and permeability, which account for the bioclogging (as it appears to some authors to be a critical point in most models) as well as the relationship between porosity and dry matter content (which accounts for the global biofilm 'structure'). In the third section, steady states profiles are then analysed in the frame of a simple steady state 1D dimensional model and some more refined features concerning the coupling between hydrodynamic and biofilm growth are discussed, in particular a possible biofilm biological response to the local flow conditions. Indeed, it appears that results obtained at the macroscopic scale cannot simply be explained if the influence of the hydrodynamic on some structural and metabolic features of the biofilm is not taken into account.

\section{Material and methods}

\subsection{Experimental setup}

The experimental setup is based on a transparent PVC column (a) which is presented in Fig. 1. Its internal diameter is $D c=15 \mathrm{~cm}$ and its height $H=67 \mathrm{~cm}$ (between the first pressure port and the 


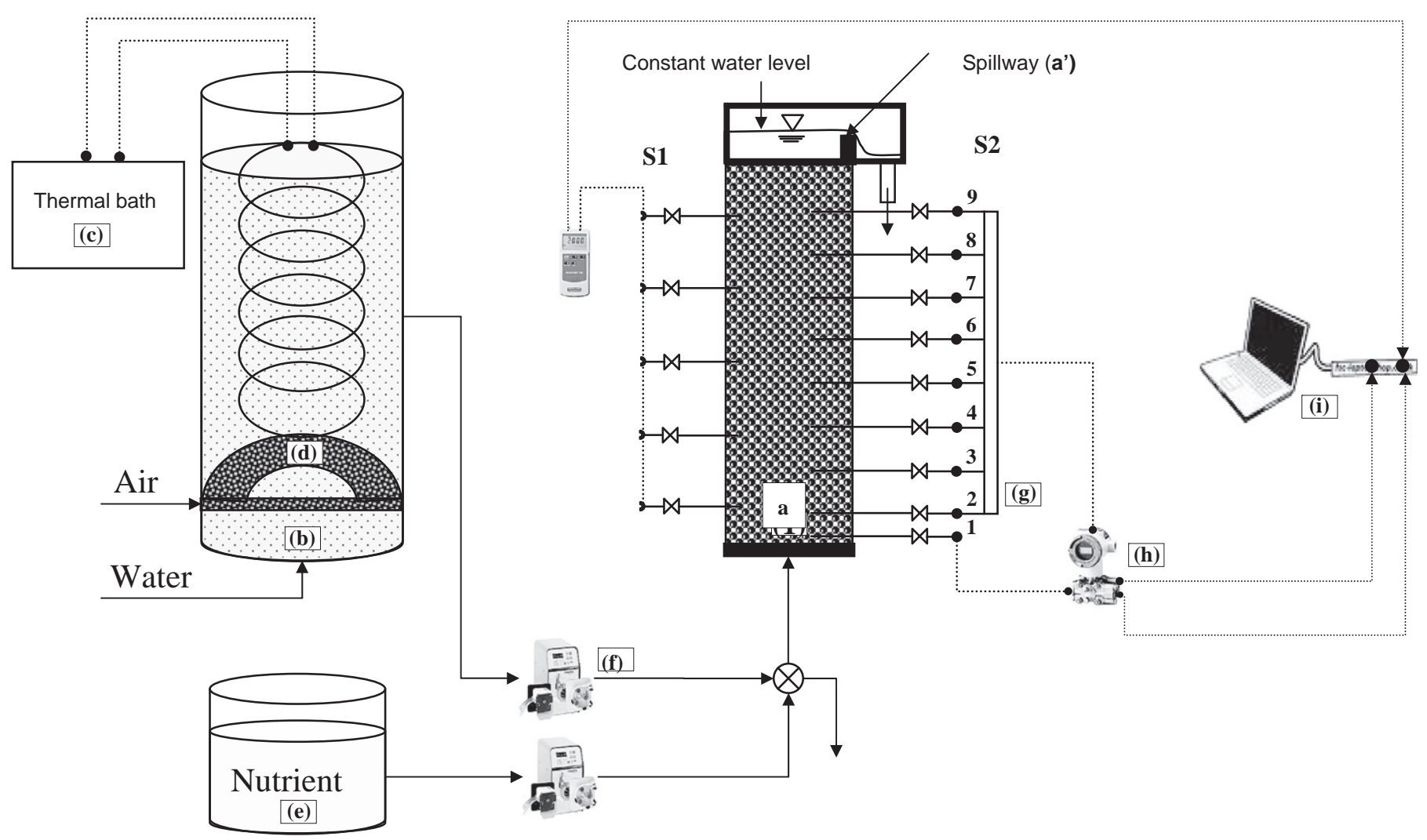

Fig. 1. Experimental setup overview.

water level surface). The column is filled with expanded clay beads (Biolite ${ }^{\odot}$, Degrémont). The Biolite ${ }^{\odot}$ beads are maintained between two grids to form a granular packed bed and to avoid a possible expansion during the experiment. The column at the top is equipped with a small tank and a spillway to ensure a water constant level surface $\left(a^{\prime}\right)$.

The beads were selected in order to ensure a size distribution as uniform as possible: their equivalent diameter is about $d_{p}=4.2 \mathrm{~mm}$ with an uniformity coefficient $d_{p 60} / d_{p 10}$ around 1.2 . The beads size distribution, presented in Fig. 2, can be then considered to be fairly monodispersed. Note that the characteristic pore size is $650 \mu \mathrm{m}$ according to the definition used in Bordas et al. (2006).

Biolite $^{\odot}$ is a commonly used carrier solid in French industrial biofilters for its high surface porosity. It was checked however that there was no risk of internal bead colonisation. The beads porosity was measured using a mercury porosimeter. Despite the high porosity of the beads ( $\pm 45 \%$ ), the pore spaces available to bacterial cells ( $>2 \mu \mathrm{m}$ ) inside the beads, represent less than $5 \%$ of the total porosity (see Fig. 3), and correspond probably to the surface rugosities of the beads. Furthermore, the figure shows clearly that the fluid-percolating step (pores that are indeed fully connected) is of $0.1 \mu \mathrm{m}$ (inflexion point), clearly lower than the size of our bacterial cells $(4 \mu \mathrm{m})$. These results confirm undoubtly that the cells cannot enter the beads and colonise the internal pore space and that the bacterial biomass grows only at the external surface of the beads as a biofilm.

The column is fed from an heated constant level water $\left(0.125 \mathrm{~m}^{3}\right)$ tank (b) in order to maintain the water temperature at $30^{\circ} \mathrm{C}$. The tank is heated using a thermal regulated bath (c). Air is continuously bubbled in that tank through a diaphragm (d) in

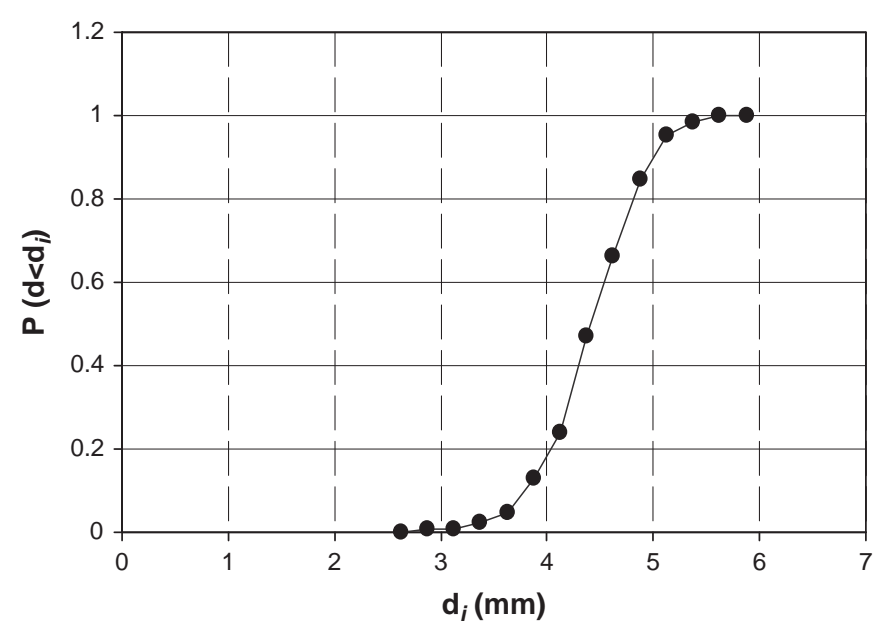

Fig. 2. Beads size distribution.

order to dissolve oxygen and maintain a constant oxygen concentration at the column inlet $\left(6 \mathrm{mg} \mathrm{l}^{-1}\right)$. A nutrient tank (e) $\left(0.025 \mathrm{~m}^{3}\right)$ contains both the phenol and the growth nutrient necessary to the bacterial growth and the biofilm development. The culture medium is a modified Lysogeny broth medium (Bertani, 1951). The original LB medium is composed with $\mathrm{NaCl}$, yeast extract and bacto-peptone, completed with distilled water. Compared to the original LB culture media, peptone was replaced with phenol. The composition for the other nutrients was $0.25 \mathrm{~g} \mathrm{l}^{-1}$ for $\mathrm{NaCl}$ and $0.25 \mathrm{~g} \mathrm{l}^{-1}$ for yeast extract which 


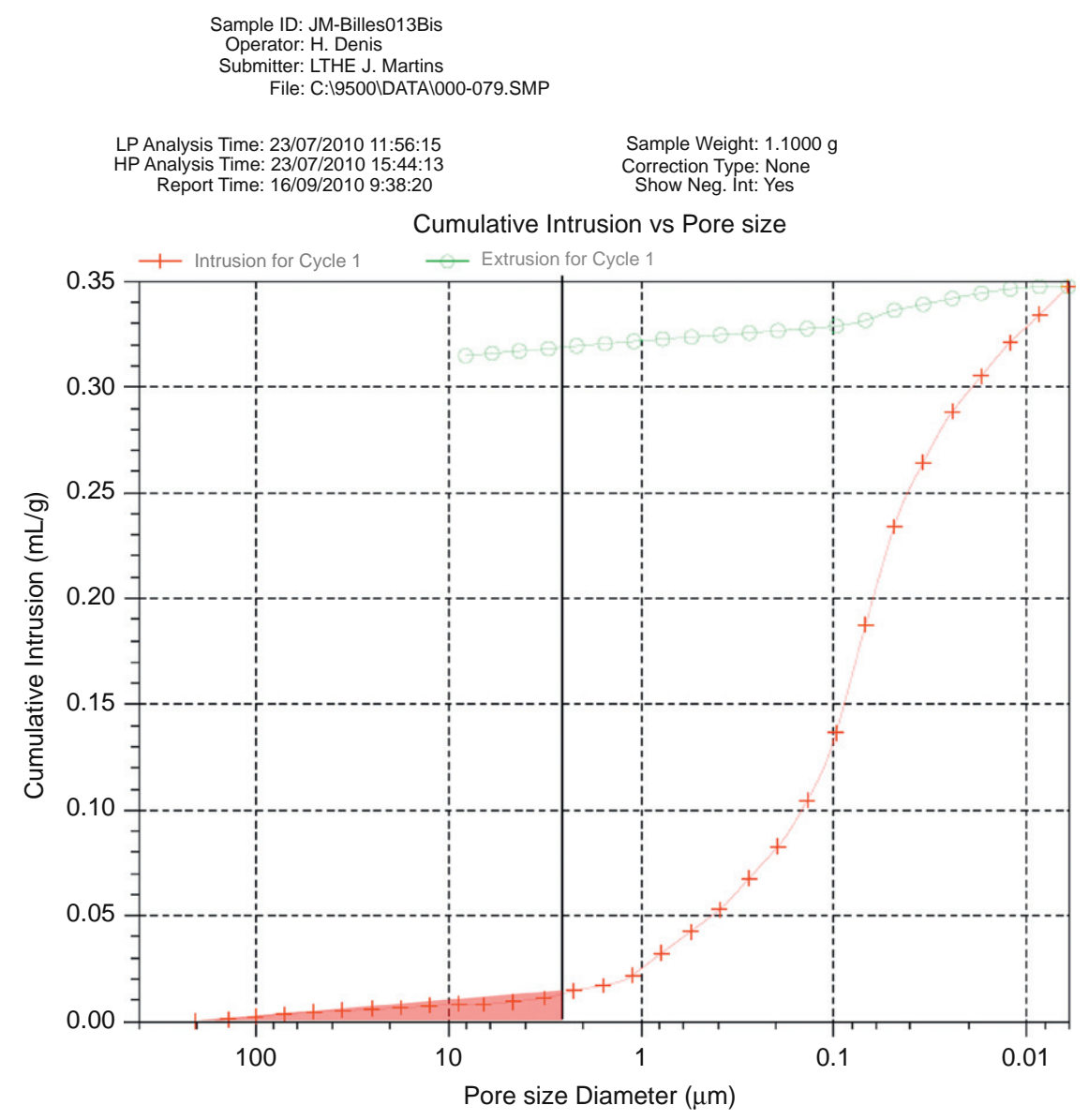

Fig. 3. Biolite ${ }^{\odot}$ pore size distribution.

correspond to a dilution factor 20 . The $\mathrm{pH}$ was adjusted to 7 and the solution sterilised in an autoclave.

The packed bed is continuously fed with peristaltic pumps (f) $\left(\right.$ Masterflex $^{\odot}$ ) connected to the two tanks. The flow rate in the bed $Q$ is the sum of the water flow issued from the main tank (b) and of the nutrient solution. The pumps were calibrated in order that a fixed roller rotating speed ensured the wished flow rate. Phenol was chosen as the carbon source and the bacterial strain used was Pseudomonas putida. In order to characterize the temporal evolution of the biofilm inside the column, the biofilter is equipped with two sampling devices composed of 5 and 9 sampling ports, respectively. The first sampling rack (S1, containing 5 evenly distributed ports) allows the sampling of the solution inside the packed bed: it is used to monitor quantities such as oxygen and phenol concentrations at different heights in the packed bed. The second rack (S2) is made up of 9 sampling ports evenly spacedistributed. These ports are connected to a multi-hole pressure tap (g) that allows the measurements of the pressure drop along the column using a single pressure sensor (Druck LPX9000) (h).

\subsection{Standard protocol of experiment}

\subsubsection{Column preparation}

The standard protocols for the granular media, substrate, culture medium and bacteria strain preparations as well as the column feeding can be found in Karrabi (2009) and Karrabi et al. (2006). They are summarised hereafter. When the column is filled with the beads, it is regularly shaken in order to achieve bed compaction with porosity as homogeneous as possible. For each experiment, the initial permeability $K_{0}$ of the packed bed is measured by varying the flow rate $Q$ and measuring the pressure drop $\Delta p_{19}$ between the pressure ports 1 located at $z=0$ and 9 located at $z=52.85 \mathrm{~cm}$. According to the Darcy's law

$U=\frac{Q}{S}=-\frac{K_{0}}{\mu} \nabla p=-\frac{K_{0}}{\mu} \frac{\Delta p_{19}}{H_{19}}$

where $U$ is the superficial velocity, $S$ the column cross section, $\mu$ the liquid dynamic viscosity and $H_{19}$ the distance between the pressure ports 1 and $9\left(H_{19}=52.85 \mathrm{~cm}\right)$ The Druck pressure sensor has an accuracy better than $0.1 \%$ full scale. Given an uncertainty about $0.1 \mathrm{~cm}$ on $H_{19}$, the uncertainty on $K_{0}$ is about $0.3 \%$. The initial porosity $\phi_{0}$, defined as the ratio between the total bed voidage and the column volume, is also measured by draining the column and measuring the drained liquid volume. The uncertainty is around $3 \%$, mainly due to the liquid static retention (Ortiz-Arroyo et al., 2003). The corresponding results taken at the beginning of each run are given in Tables 1 and 2. As it can be seen, the variability on $K_{0}$ is not negligible from one experiment to another, with a factor 2.76 on extreme values. The variability in the initial porosity is weaker, and a constant value $\phi_{0}=0.35$ is considered in the sequel. The variability of the observed value of $K_{0}$ is consistent with the observed small variations of the initial measured porosity (variations mainly due to small differences in the initial bed compaction).

The initial state of the clean column (in term of porosity and permeability) being known, after physiological adaptation of the microorganisms to phenol, the biofilter is submitted to a first stage of colonisation by $P$. putida, by percolating a culture through the column in a closed loop, during $24 \mathrm{~h}$. After this period of colonisation, the system is switched to the setup used for the biofilm growth experiments: the column is fed (in an open-loop) 
Table 1

Initial permeability for each run.

\begin{tabular}{llll}
\hline \multirow{2}{*}{ Run no. } & \multicolumn{2}{l}{ Flow rate $(\mathbf{l} / \mathbf{h})$} & \\
\cline { 2 - 4 } & $\mathbf{5}$ & $\mathbf{1 0}$ & $\mathbf{2 0}$ \\
\hline 1 & $7.3 \times 10^{-9}$ & $7.25 \times 10^{-9}$ & $1.27 \times 10^{-8}$ \\
2 & $6.93 \times 10^{-9}$ & $7.37 \times 10^{-9}$ & $1.91 \times 10^{-8}$ \\
3 & $7.15 \times 10^{-9}$ & $1.2 \times 10^{-8}$ & - \\
\hline
\end{tabular}

Table 2

Initial porosity measurement.

\begin{tabular}{llll}
\hline Run no. & \multicolumn{2}{l}{ Flow rate $(\mathbf{l} / \mathbf{h})$} & \\
\cline { 2 - 4 } & $\mathbf{5}$ & $\mathbf{1 0}$ & $\mathbf{2 0}$ \\
\hline 1 & 0.347 & 0.343 & 0.355 \\
2 & 0.355 & 0.352 & 0.358 \\
3 & 0.353 & 0.350 & - \\
\hline
\end{tabular}

by mixing water pumped from tank (b) with a concentrated nutrient solution pumped from tank (e) (see Fig. 1). The experiments presented in this paper were performed at a fixed phenol concentration of $200 \mathrm{ppm}$ at the column inlet, and for different flow rates $Q$ (namely 5,10 and $201 \mathrm{~h}^{-1}$ ).

\subsubsection{Pressure, permeability and other parameters measurement}

The pressure drop between samplers 1 and $9\left(\Delta p_{19}\right)$ is monitored online with an automat system Field point ${ }^{\mathrm{TM}}$ associated with Labview $^{\mathrm{TM}}$ software. Every day a complete pressure profile is also measured manually along the column $\left(\Delta p_{1 \mathrm{j}} ; j=\right.$ $2-9$ ). When the pressure profile is stabilized in the column (no significant variation of the mean pressure profile during 3 days), it is considered that a steady state is achieved. The measured pressure drop in each layer is then used to deduce the layer permeability $K(z)$ from Darcy's equation with an uncertainty about $11 \%$. When a pressure profile is manually measured, the first rack containing the five sampling ports may be used to collect effluent samples and to measure various quantities: in particular, it was initially planned to measure both the oxygen and the phenol concentration profiles and their evolution with time. However, in our operating conditions, the phenol degradation appeared to be quite small (less than $25 \%$ at the column outlet for the best case). As the available measurement procedure for the phenol was a colorimetric method, and because of this small amount of degradation, the uncertainties on the measured resulting phenol concentration would be prohibitive in order to give accurate results on the evolution of the phenol concentration profiles with time. That is why it was not systematically recorded. Yet, the global consumptions of phenol were measured at the end of some experiments. On the opposite, the oxygen profiles were systematically measured when a pressure profile was manually measured (the protocol is presented below).

\subsubsection{Oxygen measurement protocol}

Liquid samples $(10 \mathrm{ml})$ were removed from various heights of the bed using a syringe and without any contact with air. The liquid was then poured into a specific vessel and oxygen concentration was measured immediately using a Clark DO probe (whose diameter is very close to that of the vessel). The sampling time was far shorter than the characteristic time for $\mathrm{O}_{2}$ consumption in the column and each sampling can be considered as representative. The tube was closed hermetically with cotton in order to limit air entry. The measurements were performed until an equilibrium value was reached $(\sim 1 \mathrm{mn})$. The probe response time is $16 \mathrm{~s}$ to reach $90 \%$ of the final value and less than $1 \mathrm{mn}$ for $99 \%$ of the final value. Using this very "classical" protocol, we obtained very reproducible values.

\subsubsection{Porosity and biomass profiles measurement}

The column is divided into 6 layers (each about $12.5 \mathrm{~cm}$ high) delimited by the sampling ports used for the oxygen and phenol measurements (see Fig. 1). When the experiment is stopped, each layer is carefully drained at a very low flow rate, through each sampling port. For some experiments, the collected water volume was used to compute the mean (mobile) porosity $\phi(z)$ in each layer. When a layer is drained, the corresponding colonized Biolite $^{\circledR}$ is recovered. The biofilm of each layer is extracted by vigorous mechanical agitation $(200 \mathrm{rpm})$ in 21 of distilled water during $15 \mathrm{~min}$. The same washing method is applied to each Biolite $^{\circledR}$ layer, in order to recover the superficially attached biomass. After beads sedimentation, the supernatant containing the biomass is collected and stored at $4{ }^{\circ} \mathrm{C}$ before performing qualitative and quantitative analysis within $24 \mathrm{~h}$. The total dry mass $m_{x}$ is calculated by drying $100 \mathrm{ml}$ of the samples at $105{ }^{\circ} \mathrm{C}$ during $24 \mathrm{~h}$. The quantity $c_{x}$ is then defined as the total dry matter over the initial void volume $v_{i v}$ in a layer

$c_{x}=\frac{m_{x}}{v_{i v}}$

This quantity will be referred to as the biomass concentration in the sequel and it corresponds to the dry mass of "EPS + microorganism" in the sample.

Let us notice that the column geometry at the exit prevented a continuous survey of the biomass concentration at the outlet. The water volume above the porous media is submitted to some mixing. Furthermore, as it will be seen later, the exiting biomass is not only composed of free cells but also sometimes of biofilm fragments (cell-EPS aggregates). The detached biomass floats at the free surface, and only part of it is evacuated over the spillway.

\subsubsection{Controlled parameters and operating range compared to existing experiments}

The controlled parameters are the flow rate $Q$ the inlet substrate concentration $c_{s 0}$, the water temperature $T$ and the inlet dissolved oxygen concentration $c_{o i}$. Table 3 compares our experimental operating conditions to existing experiments in terms of non-dimensional numbers. The operating conditions lie in the range of the operating conditions performed in previous experiments found in literature (see Table 3) (Darcy Regime, moderate Damkohler number) with slightly higher Peclet numbers. Table 3 gives also other parameters such as the couple bacteria strain/ carbon source, the characteristic pore size and, when available, the organic load (expressed as $\mathrm{kg}$ COD/day)

\section{Experimental results}

\subsection{Experimental conditions assessment}

Before presenting the data, it is important to ascertain a number of characteristics of the flows considered. In particular, the analysis we will present in the following relies on the assumption that the Darcy law is valid in all our experimental conditions. We therefore have to check whether the fluid properties of the interstitial liquid are changing and whether the flow regime remains laminar during the course of the experiments. In addition, it is also important to determine the initial porosity as well as the initial permeability. 


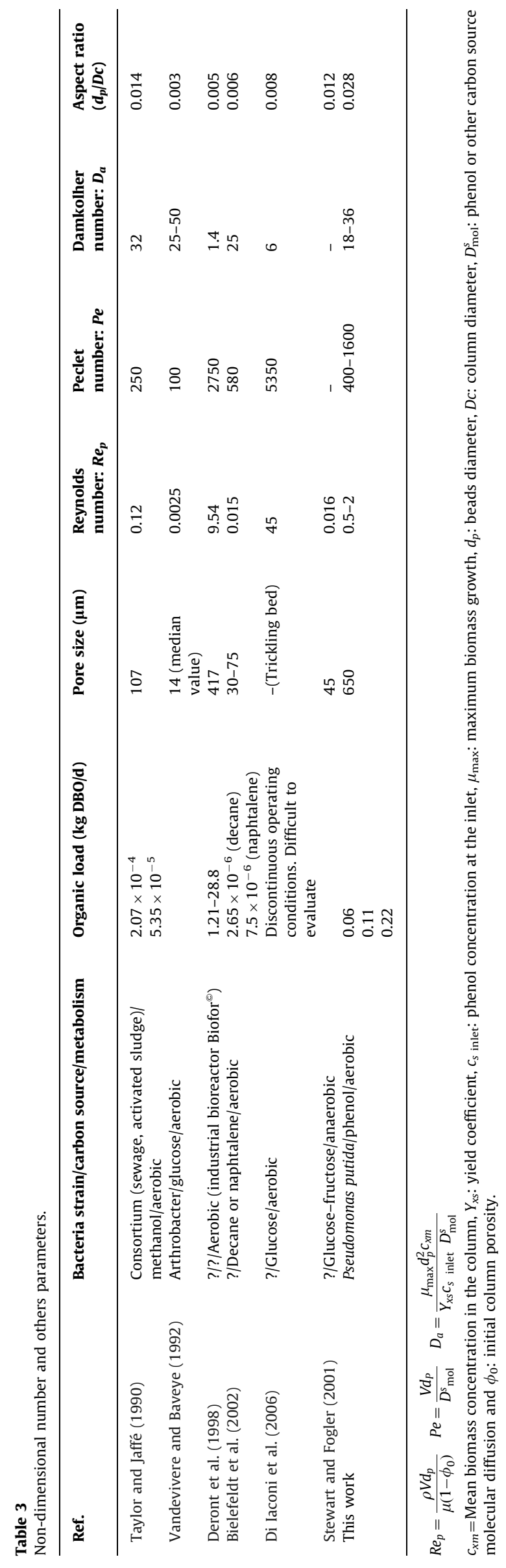

\subsubsection{Fluid properties}

As the biofilm develops and polymeric substances are produced, the interstitial fluid properties may change during the course of the experiment. An interstitial fluid sample was taken at the bottom of the column (the part which was the most clogged) for one experiment. This sample was analysed using a rheometer. ${ }^{1}$ No deviation from a Newtonian behaviour was observed for shear rates ranging from 1 to $1000 \mathrm{~s}^{-1}$. Viscosity measurements where then performed on interstitial liquid samples taken along the column during a typical experiment. No significant viscosity changes were observed. In the sequel, a constant viscosity $\mu$ equal to that of water will be therefore considered.

\subsubsection{Flow regime for the clogged column}

The validity of the Darcy's law was checked for the clean column when the initial permeability was measured (see Section 3.2). The data analysis procedure requires that this law remains valid for the clogged column. This task is not straightforward without the knowledge of the biofilm distribution within the pore which influences the local flow field. Indeed, the classical definition of the Reynolds number as well the classical critical value for the departure from Darcy's law, are derived for systems with a fixed geometry. Here, we consider the following definition of the pore Reynolds number

$R e_{p}=\frac{\rho_{f}(Q / S) d_{p}}{\mu(1-\phi)}$

where $Q$ denotes the flow rate, $S$ the area of the entire section of the column, $d_{p}$ the colonized beads diameter, $\phi$ the column mean porosity in a layer between two sampling ports. $R e_{p}$ was compared with the usual critical value of 10 that indicates the frontier of the laminar regime. In that scope, an estimate of the evolution of the colonized beads diameter with porosity, $d_{p}(\phi)$, has been computed under the geometrical assumption of a uniform bead colonisation (using a procedure close to Taylor and Jaffé, 1990) and a regular beads arrangement (body centred cubic). In addition, we used the porosity measurements (presented in the next paragraph) to estimate $R e_{p}$. It happens that for the most plugged conditions which also correspond to the largest flow rate $\left(20 \mathrm{l} \mathrm{h}^{-1}\right)$, the Reynolds number always remains below 1.8 (Karrabi, 2009). Despite the assumption made on the biofilm structure at the pore scale, it is likely that the $R e_{p}$ never reaches 10. Therefore the flow remained laminar in all the conditions considered, and Darcy's law is applicable.

\subsubsection{Initial conditions (initial biomass repartition)}

Between two experiments performed during the same measurement campaign, the beads were always cleaned following the same protocol (cf. Section 2.2.4). The beads were then sterilised in an autoclave in order to inactive the (possible) remaining attached biomass. The beads were then ready to be used in the next experiment. This way all the precautions were taken to ensure as much as possible the same initial conditions at the start of each experiment.

\subsection{Raw data presentation}

\subsubsection{Existence of a steady state}

The first question that deserves to be addressed concerns the existence of a steady state. Fig. 4 shows the time evolution of the pressure drop in the biofilter for three flow rates. Note that the data for $Q=51 \mathrm{~h}^{-1}$ were collected manually while for $Q=10$ and $201 \mathrm{~h}^{-1}$, they were continuously monitored at a sampling

\footnotetext{
${ }^{1}$ NRc2, Laboratoire de Rheologie (UMR, Grenoble).
} 


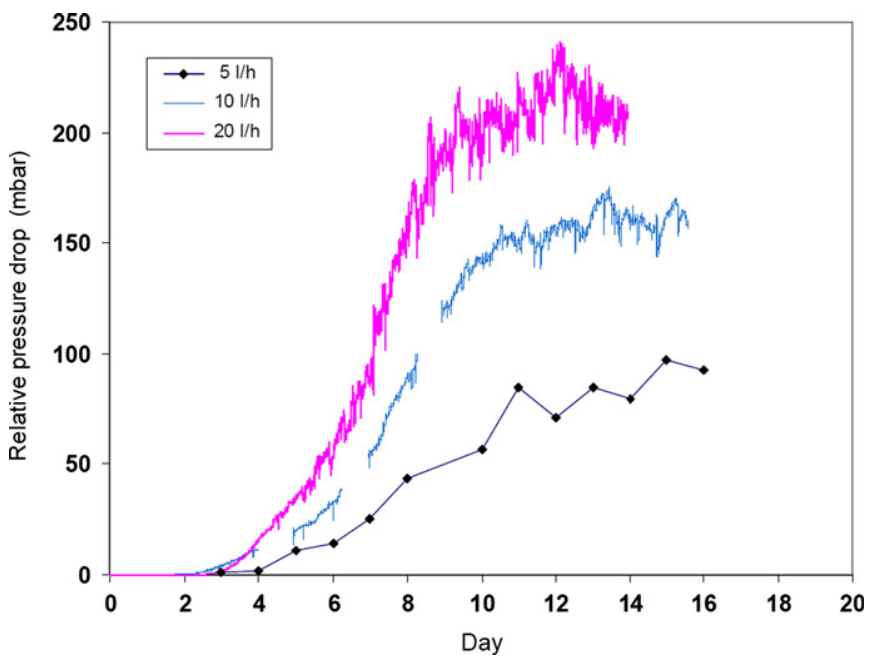

Fig. 4. Time evolution of the pressure drop in the column for the 3 flow rates and for the same measurement campaign.

rate of $0.0083 \mathrm{~Hz}$ ( 30 points $/ \mathrm{h}$ ). Time $t=0$ corresponds to the beginning of an experiment, when the system is switched from the colonisation closed-loop circuit, to the open-loop nutrient+ water circuit. After a lag time (typically 2-3 days) that corresponds to the physiological adaptation of the bacteria to the chemical conditions, the pressure exhibits a progressive and significant increase. Such a behaviour is linked with the bacterial growth and/or the EPS production: as the biofilm develops around the granular clay balls and/or within the bioreactor pores, the porosity of the packed bed is reduced. That reduction is important as shown by the typical values of the pressure drop which are tens to hundreds times larger than the pressure drop for the clean column (the latter is about 0.1 mbar). The last stage corresponds to the stabilisation of the pressure drop around mean levels which are about 90,150 and 210 mbar for flow rates equal to 5, 10 and $20 \mathrm{l} \mathrm{h}^{-1}$, respectively. These steady states correspond to a "global" equilibrium between processes such as the cells division, cells and biofilm detachment and eventually recapture that are mainly controlled by local hydrodynamic conditions (shear stress), substrate and oxygen availability (growth inhibition), biofilm structure (thickness, adhesion, yield stress, etc.). Let us notice that, as the fixed bed is blocked between two grids, the pressure level increase cannot lead to the bed fluidisation and to a sudden loss of biomass by a complete sloughing of the bioreactor.

Oscillations around the mean pressure drop levels are also present. Fig. 5 corresponds to a zoom on the pressure curves corresponding to the 10 and $20 \mathrm{l} \mathrm{h}^{-1}$ flow rates. Oscillation amplitudes around the mean pressure drop are around \pm 20 mbar in both cases. Such a behaviour was also observed by Stewart and Fogler (2001). In their case, the packed bed was $5.75 \mathrm{~cm}$ long for a diameter of $2.5 \mathrm{~cm}$. It was filled with $300 \mu \mathrm{m}$ glass beads (giving a characteristic pore size around $45 \mu \mathrm{m}$, see Table 3). Their packed bed geometry is such that the observed mean pressure is around $1000 \mathrm{kPa}$ with oscillations amplitudes around $\pm 1000 \mathrm{kPa}$ ! These authors explained these oscillations by different processes which interact. In particular, the authors interpret the oscillations as both growth and polymer production combined with hydrodynamic redistribution of biomass due to biofilm sloughing and mechanical recapture downstream. The biofilm sloughing would also create channels within the plugged part of the porous media, which influence the nutrient flow and dispersion within the column.

In our case, the oscillations amplitudes are less important, partly because of our larger pore dimension. We defined a
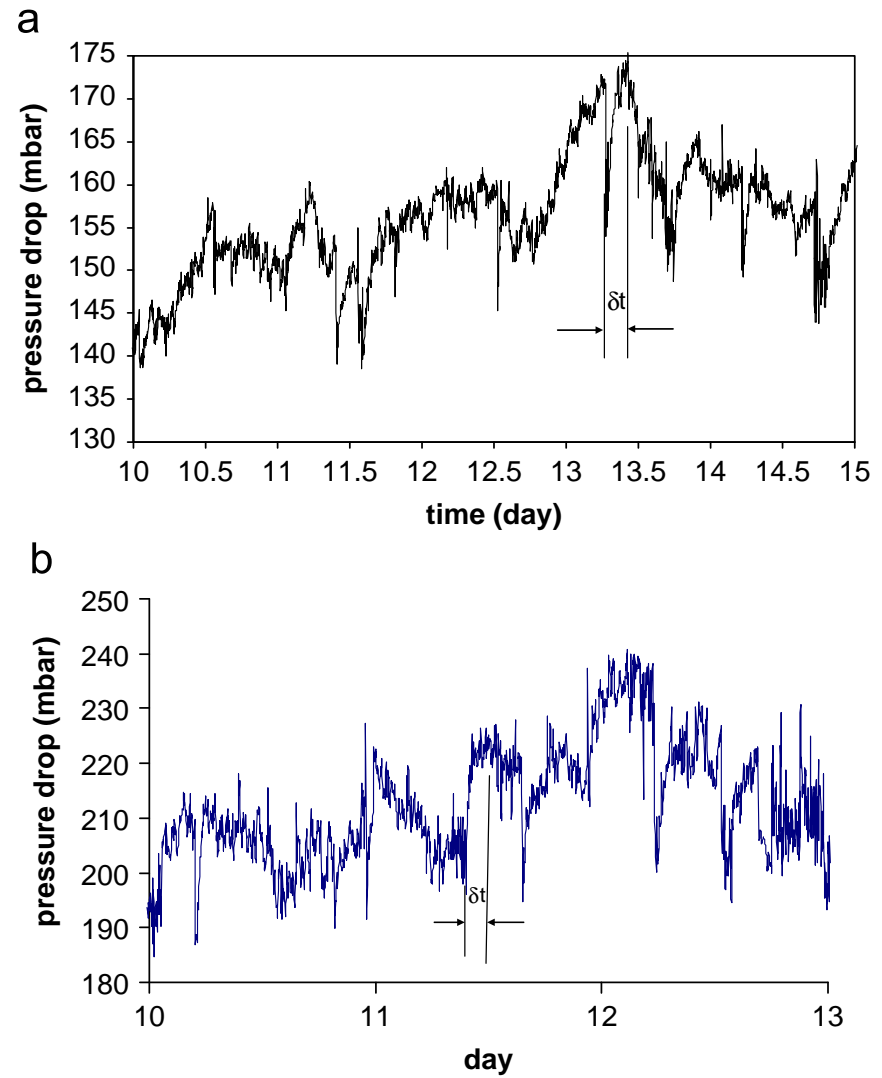

Fig. 5. Typical time scale of pressure amplitudes oscillations.

characteristic time scale $\delta t$ as the time between a local minimum and maximum on the pressure drop curve (Fig. 5). It is found that this characteristic time is about $3.5 \mathrm{~h}$ for $Q=10 \mathrm{~h} \mathrm{~h}^{-1}$ and $2.5 \mathrm{~h}$ for $Q=201 h^{-1}$. These values are too high to be attributed to a phenomenon of sloughing with recapture downstream within the column. Moreover, Hill and Robinson (1975) report values of the bacterial maximum growth rate for $P$. putida ranging from $1.5 \times 10^{-5}$ to $1.5 \times 10^{-4} \mathrm{~s}^{-1}$ i.e. a characteristic time scale about 1.85-18.5 h. Given the discrepancies on the available data about this parameter, the bacteria typical time scale is of the same order as the time scale of the high amplitude oscillations observed on the pressure curves. This would favour an explanation where local biomass growth (or perhaps polymer production) compensates for biofilm detachment (detachment and recapture would occur on a far shorter time scale).

\subsubsection{Pressure profiles}

Fig. 6 shows the transient pressure profiles corresponding to Fig. 4. The profiles were deduced from pressure drop measurements using Eq. (4) where $P_{i+1}$ is the pressure at the pressure port $i+1, \Delta P_{i, i+1}$ is the pressure drop measured between port $i$ and $i+1$, and $\Delta z_{i+1}$ is the distance between pressure port $i$ and $i+1$. The reference pressure was set to zero at the water level surface and the pressure drop was neglected to compute the pressure corresponding to needle no. 9 (which is valid as most of this part of the column is not filled with beads). We observe that the pressure profile at the start almost correspond to the hydrostatic pressure profile, as, for the clean packed bed, the pressure loss is very weak for our operating conditions (flow rate and beads size). This can be checked using the Darcy law which links the initial permeability to the bed geometry

$P_{i+1}=P_{i}+\Delta P_{i, i+1}+\rho g \Delta z_{i, i+1}$ 
a

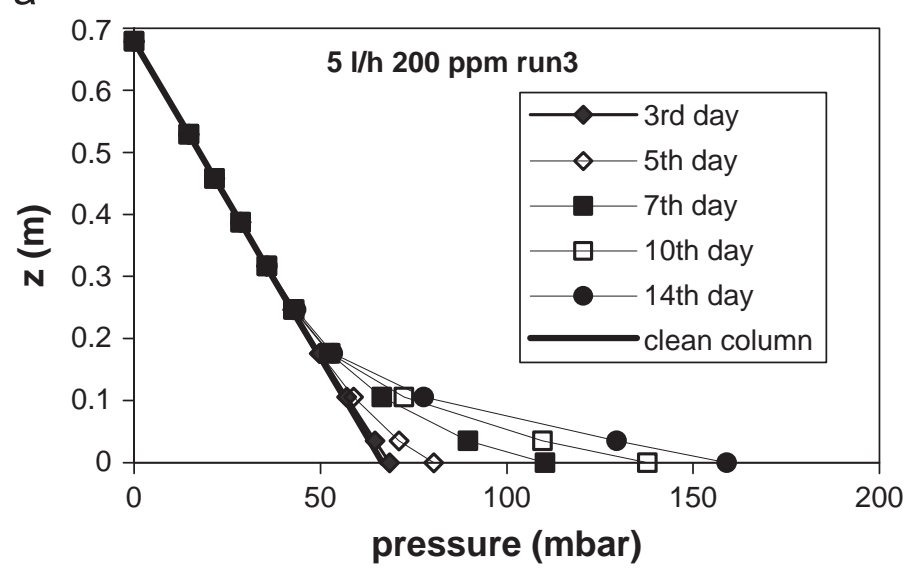

C

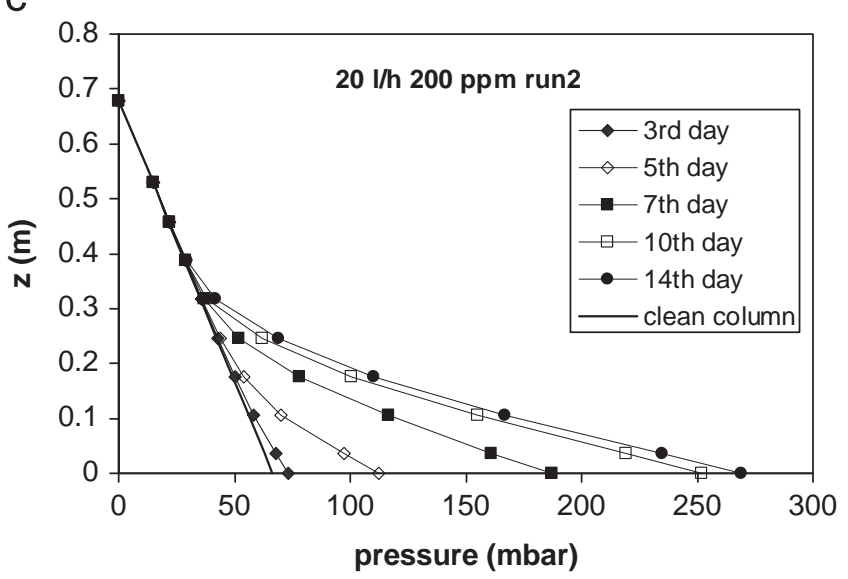

b

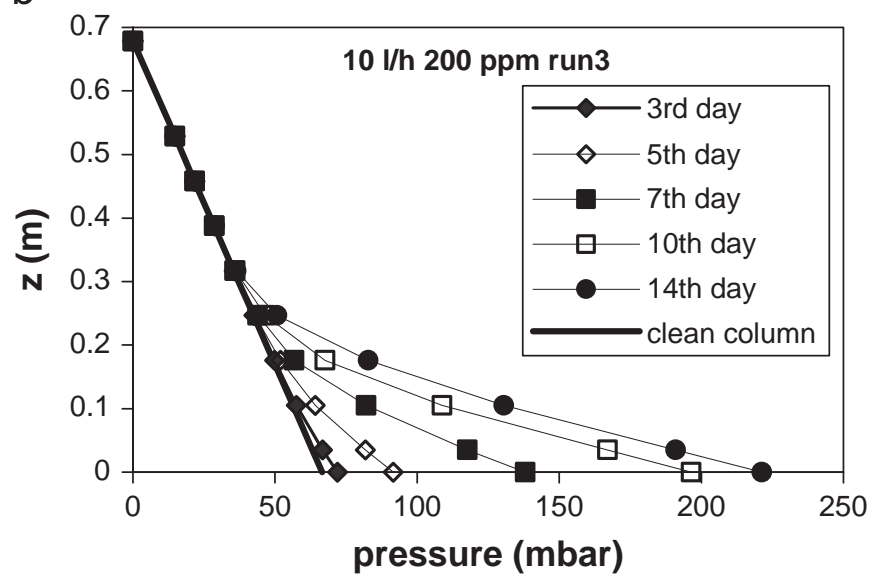

d

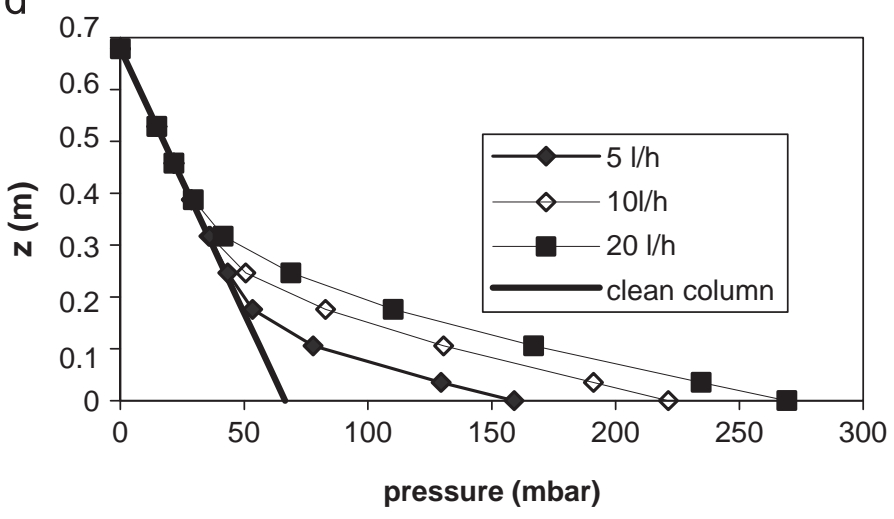

Fig. 6. Pressure profiles measured across the biofilter for different flow rates. (a) $Q=51 \mathrm{~h}^{-1}$, (b) $Q=101 \mathrm{~h}^{-1}$, (c) $Q=201 \mathrm{~h}^{-1}$ and (d) steady state conditions.

The biofilm growth starts at the column bottom, where the nutrient is available first, and the pressure begins to increase as the pore space begins to be clogged. The position within the column where the pressure profiles are again linear corresponds to the clogging front location. This clogging front moves inside the column until it reaches a steady position ranging from $1 / 3$ to $1 / 2$ of the column height.

The existence of a steady state as reported here was not always observed: on an industrial scale reactor, Deront et al. (1998) observed the same behaviour as in our experiment contrary to Stewart and Fogler (2001) who observed a complete plugging on a micro-scale packed bed. As Deront et al. (1998) experiments where performed in an aerated biofilter and Stewart and Fogler (2001) experiments with bacteria in a state of starvation, this can be explained by the biofilm structure under different feeding conditions; this would affect its resistance to the flow, the production of detached fragment and their nature (size, composition).

This observation indicates that if a detachment process exists in our operating conditions, it does not seem to have an influence on the clogging front localisation. This fact, along the characteristics time scales discussed in the previous paragraph would indicate that the detached biomass fragments are not captured deeper in the biofilter, and that the axial biomass repartition is mainly controlled by local growth in our conditions.

These profiles allow also to more precisely define the notion of steady state, and to justify our choice to stop the experiments around the 15th day. Indeed, it can be seen that the pressure profiles do not evolve much between the 10th and 14th day compared to the evolution observed between the 7th and 14th day. This behaviour was the same at 10 and $20 \mathrm{l} \mathrm{h}^{-1}$. That is why, for the experiments presented here, the criteria chosen to stop the experimentation (no mean pressure change for 3 days) is acceptable. This point will be confirmed in the next paragraph when the experiments reproducibility will be checked.

\subsubsection{Steady states reproducibility}

The steady states reproducibility can be checked through various parameters.

In order to check the pressure drop reproducibility, another set of experiments was performed over a longer duration. For those experiments, only the total pressure drop was measured (manually). It is seen (Fig. 7) that the transient phase exhibits some changes from a measurement campaign to another (lag time possibly due to different initial biomass distributions). However, (i) the overall transient duration from one campaign to another remains nearly the same, and (ii) the existence and the reproducibility of the steady states is ensured as the pressure levels are the same as in Fig. 4. This means that the protocols used to prepare the bacteria strains, to colonise the column and to perform the experiments allow a well controlled behaviour of the column behaviour at given operating conditions.

The pressure and biomass profiles reproducibility was also checked. Fig. 8 (left side) presents the pressure profiles at steady state for different runs performed under the same operating conditions. The reproducibility of the pressure drop profiles is quite good for 10 and $20 \mathrm{l} \mathrm{h}^{-1}$. For the lowest flow rate, the 

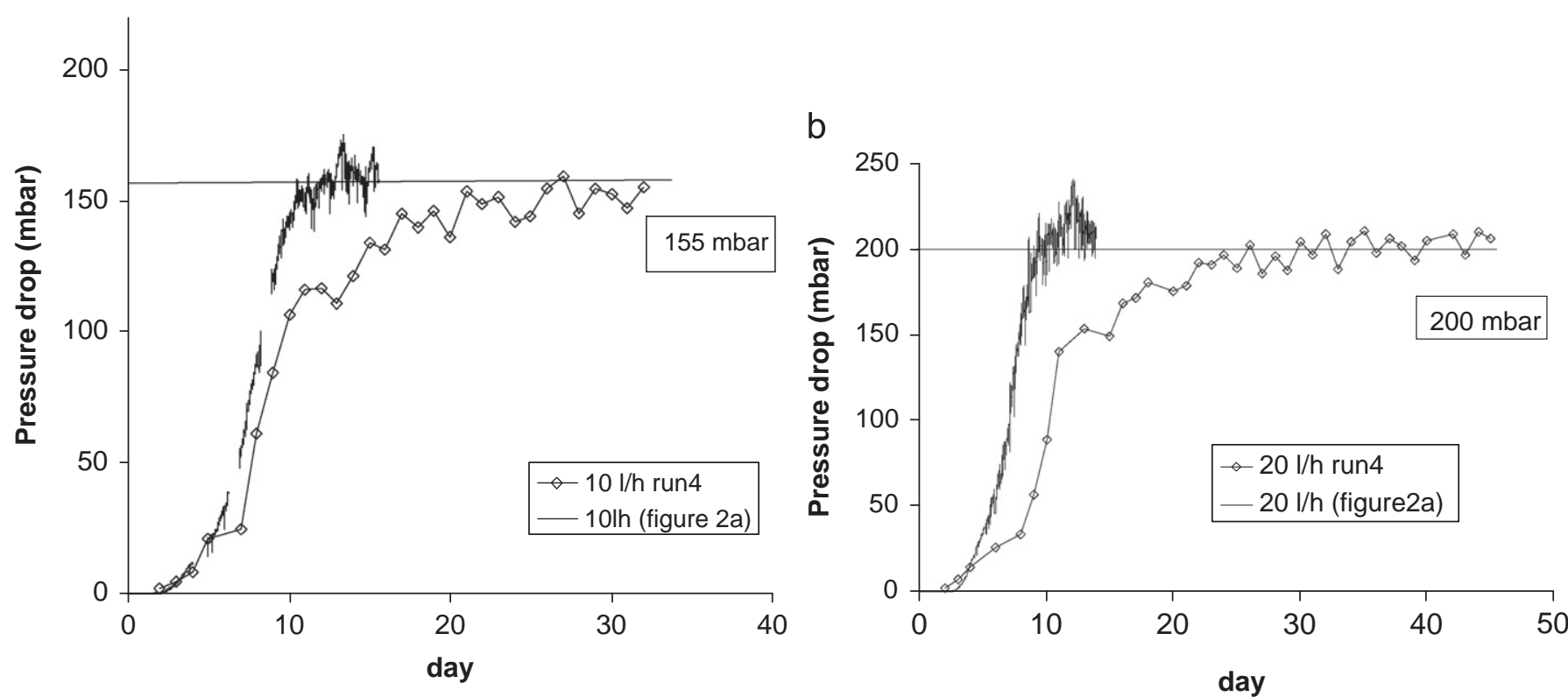

Fig. 7. Total unsteady pressure drop for different initial conditions. (a) $101 \mathrm{~h}^{-1}, 200 \mathrm{ppm}$ and (b) $201 \mathrm{~h}^{-1}, 200 \mathrm{ppm}$.

reproducibility is less obvious. This can be explained from the temporal evolution of the pressure curve: as already said, at steady state, there exist oscillations around the mean pressure level. Their relative amplitude compared to the mean pressure drop level is higher at $5 \mathrm{lh}^{-1}$. Depending on the time when measurements were taken, this can lead to some discrepancy on the pressure profiles. In the sequel, we will refer to Run 3 for the experiment at $51 \mathrm{~h}^{-1}$.

The same analysis was performed with the biomass concentration profiles to check the consistency of the data and to validate the measurement procedure presented in previous section. These profiles are also presented in Fig. 8 (right side). As the number of effluent sampling port is less than the number of pressure ports, the results concerning the biomass concentration and the porosity have been interpolated on the 8 locations for which the permeability and the pressure drop were measured.

As it can be seen from this figure, taking into account the measurements uncertainties ${ }^{2}$ the biomass concentration profiles does not show a great variability at 10 and $201 \mathrm{~h}^{-1}$. The discrepancy on the concentration at $5 \mathrm{l} \mathrm{h}^{-1}$ is in accordance with the measured pressure profiles as the pressure drop is directly related with the biomass content.

As a conclusion, raw data show that a steady state is reached for each experiment. The experimental conditions also appear to be sufficiently well controlled to ensure that this steady state is reproducible, for each studied flow rate. It is then possible to investigate the coupling between global variables from these steady conditions. This is the purpose of the next paragraph.

\subsection{Data analysis}

\subsubsection{Relation between biomass concentration and porosity}

At the steady states, the biomass concentration profiles and the porosity profiles have been measured according to the

\footnotetext{
${ }^{2}$ They were estimated to $10 \%$ at most: they include uncertainties on the layer volume when the column is emptied layer by layer, uncertainties on the total mass recovered in a layer during the procedure of dilution in the 21 of distilled water, then uncertainties on the volume sample and weighting to compute the concentration.
}

procedure presented in Section 3.2 (Fig. 9(a) and (b)). The corresponding measured oxygen profiles (which will be used later in Section 3) are presented in Fig. 10. As said before, the profiles of phenol concentration were not measured. However, the phenol concentration at the column outlet was measured at the end of the experiments. The phenol concentration were 175 , 160 and $125 \mathrm{mg} \mathrm{l}^{-1}$.

From the biomass and porosity profiles given in Fig. 9, it is possible to deduce a relation between the biomass content and the column porosity (Fig. 11). The data come from three independent experiments and lead to a fairly simple relationship between the porosity and the biofilm volumetric density (taken as the amount of dry biomass per volume of wet biofilm, according to Peyton (1996) definition).

This quantity can be measured as follow. The porosity in each layer is connected to the volume of drained water $v_{d w}\left(\mathrm{~m}^{3}\right)$. The hydrated (wet) volume of biofilm, $v_{x}\left(\mathrm{~m}^{3}\right)$, results from the difference between the initial void volume, $v_{i v}\left(\mathrm{~m}^{3}\right)$, and the drained water volume for each layer, assuming that the initial porosity of each layer is equal to $\phi_{0}$ the mean column porosity.

$v_{x}=v_{i v}-v_{d w}$

As the dry biomass, $m_{x}(g r)$, is also quantified for each layer, the biomass concentration, already defined by Eq. (2) can be computed.

The ratio between the dry biomass and the volume occupied by the wet biofilm in a layer determines the volumetric mass density of the biofilm

$\rho_{x}=\frac{m_{x}}{v_{x}}=\frac{m_{x}}{v_{i v}-v_{d w}}=\frac{m_{x}}{v_{i v}\left(1-v_{d w} / v_{i v}\right)}=\frac{c_{x}}{\left(1-\phi(z) / \phi_{0}\right)}$

As the plot $\phi=f\left(c_{x}\right)$ is linear, from Fig. 11, the volumetric density $\rho_{x}$ can be deduced from the slope and we get $\rho_{x}$ equals $25 \mathrm{~g} \mathrm{l}^{-1}$. Peyton (1996) reports biomass volumetric densities from different authors ranging from 10 to $130 \mathrm{gl}^{-1}$. Our value is in particular consistent with values found by Garcia Lopez et al. (2003) for low Reynolds numbers. The result above shows that, for our operating conditions - namely high phenol concentration and low Reynolds number - it seems that there is not a great change of the mean biofilm organisation along the column: at the 

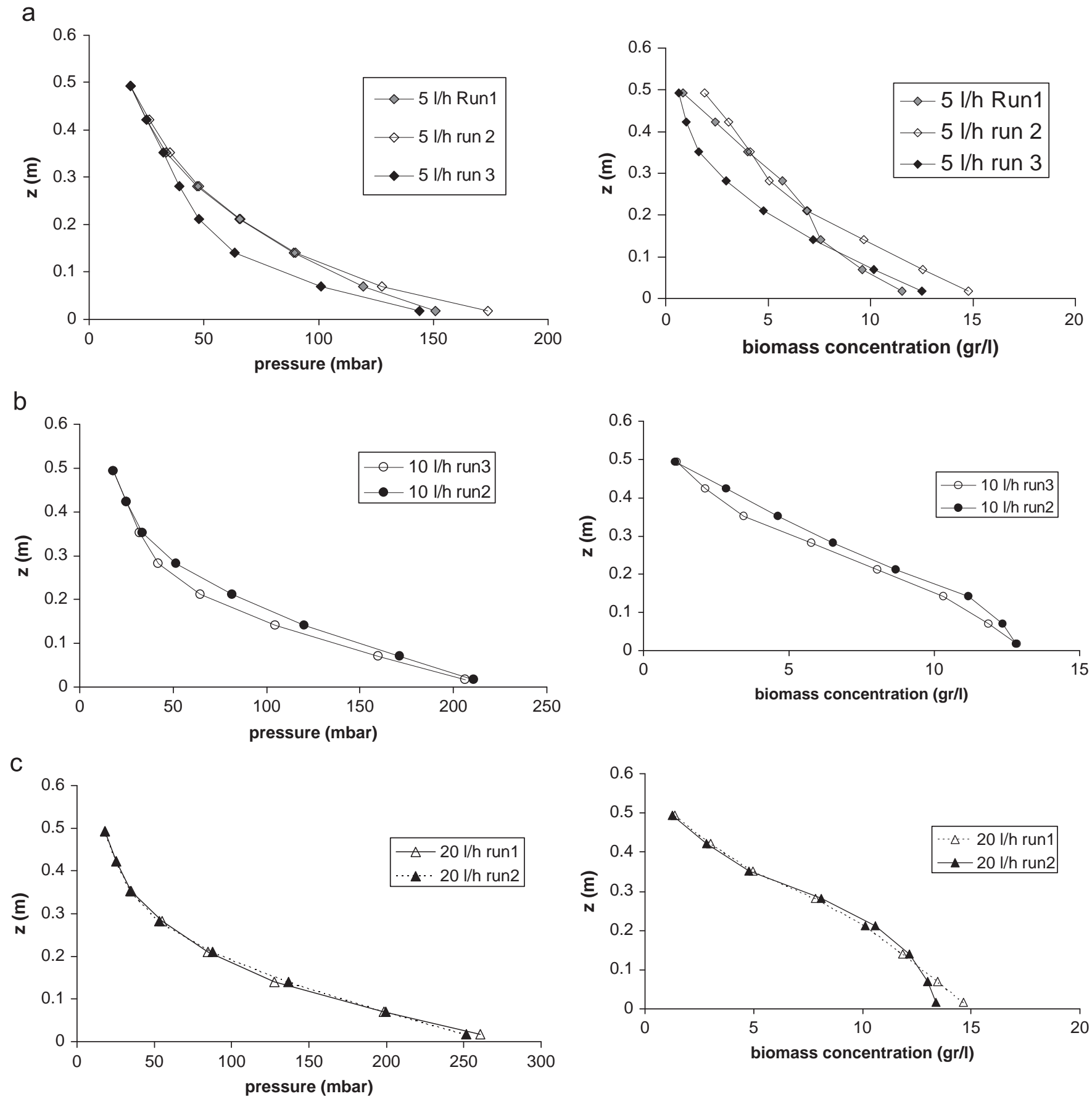

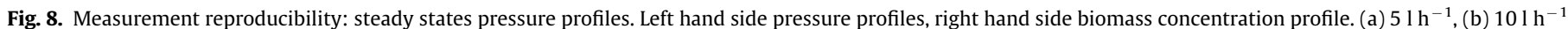
and (c) $201 \mathrm{~h}^{-1}$.

steady state, the relationship $\phi\left(c_{x}\right)$ is linear for different operating conditions, leading to a constant volumetric density. Let us notice that this observed linearity between the biomass content and the porosity may be due to the slow hydrodynamical conditions which favour the development of a heterogeneous and not very dense biofilm (Van Loosdrecht et al., 2002) along the column. The observed linearity must not be taken as a general behaviour: the task of deriving a general model about the relationship between the biomass content and the porosity requires a deep knowledge of the biofilm structure and biomass distribution. It is not possible yet from global measurement to deduce this microstructure nor the biomass distribution (at least with the method used in this paper). As for the permeability-biomass content relationship (see later), generalisation of some features observed here requires more experiments on a wider range of parameters and on both scales (macro and micro).

\subsubsection{Relation permeability-biomass concentration}

The pressure profiles and the porosity profiles at the steady state allowed the derivation of Fig. 12(a) which represents the evolution of the relative column porosity and the relative permeability in each column slice.

The reduction of permeability is very sharp as soon as the biofilm appears. This sharp decrease is commonly accredited to the existence of biopolymers (EPS) but the biofilm shape may 
a

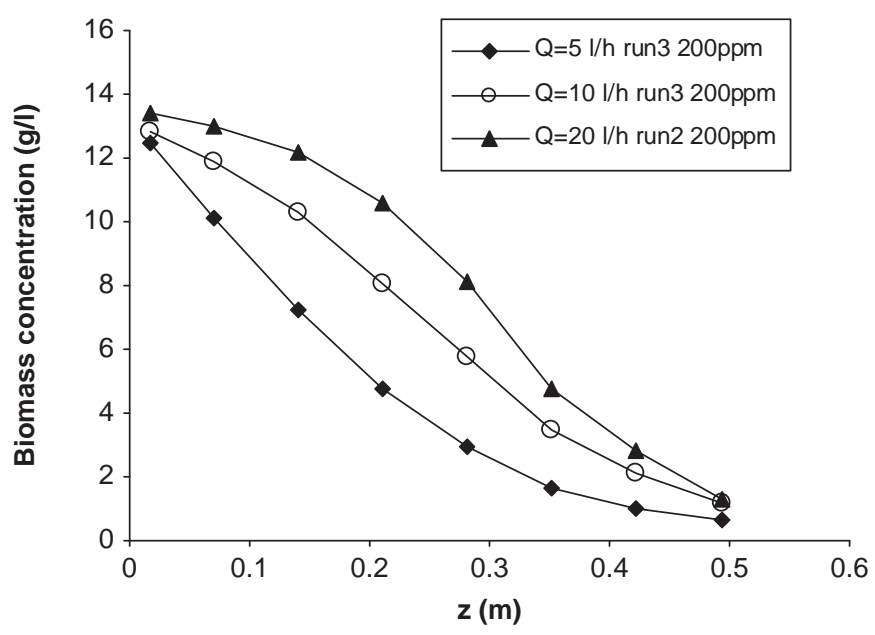

b

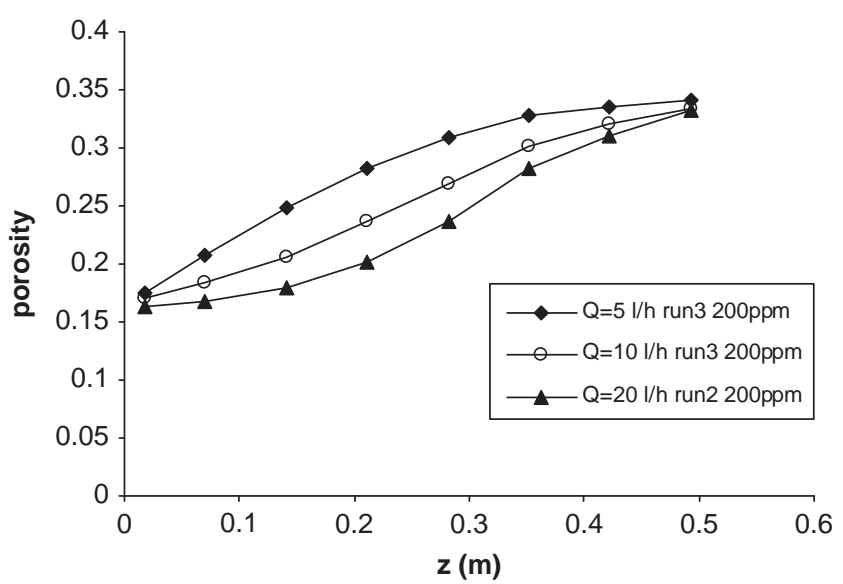

Fig. 9. (a) and (b): biomass concentration and porosity profiles at the steady state.

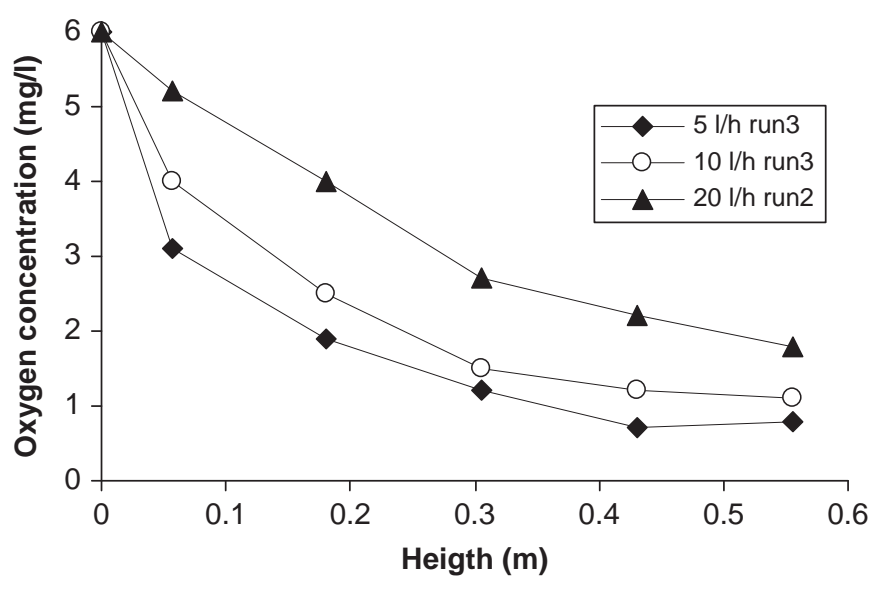

Fig. 10. Oxygen profiles at the steady state.

have also its importance. Concerning the EPS, their importance has already been stressed by Shaw et al. (1985): these authors performed clogging experiments using living or dead cells. A clear behaviour difference was observed between living cells and dead cells, the later behaving like inert colloids. These authors stated that EPS were responsible for their observations as they noticed that structures built at the pore scale by living and dead cells were quite different and those structures involved EPS. Those microstructures had a clear influence on the sharpness of the

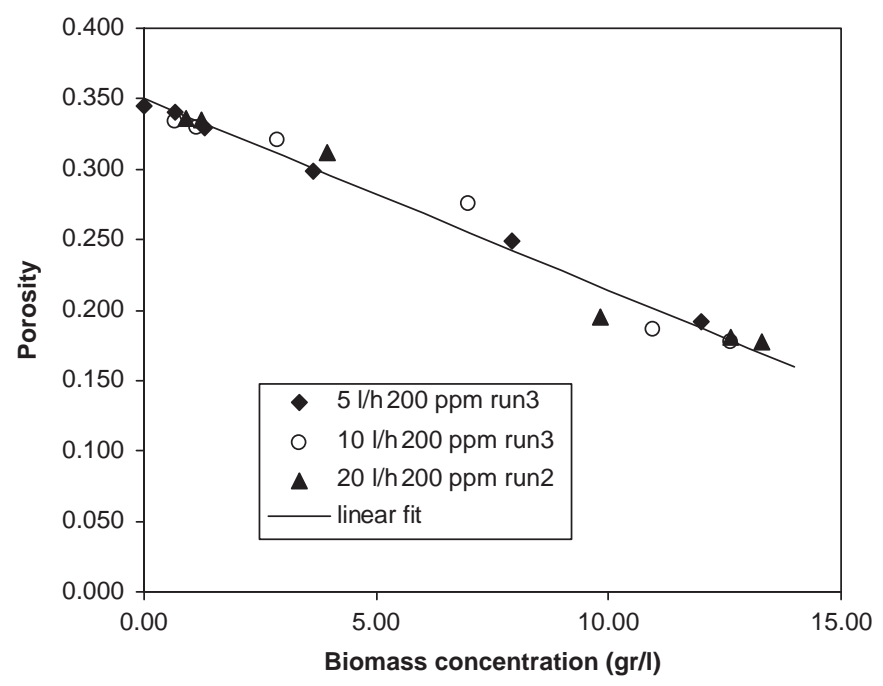

Fig. 11. relationship between the porosity and biomass concentration.

permeability decrease. The exact role of EPS on the permeability reduction and how the EPS, the biofilm structure and the hydrodynamic are related is still an issue. As stated in the previous paragraph, the hydrodynamical conditions have a strong influence on the biofilm microstructure, from strongly heterogeneous one at low Reynolds number to thinner, more compact with a less tortuous interface at higher Reynolds number (Van Loosdrecht et al., 2002). This shape affects the external and internal flow and may affect the pressure loss through eddy dissipation. It is not clear how the EPS affects these biofilm structures however. For instance Vandevivere and Baveye (1992) observed very different EPS matrix structure for biofilm grown under the same conditions in porous media, but with different bacteria strain. Another feature, concerns the existence of biofilms showing filamentous microorganisms. Siegrist and Gujer (1985) discussed how these filamentous bacterias could protude from the biofilm in the mobile fluid zone, disrupting the flow, increasing the friction surface and causing eddy diffusion. This filamentous growth is maybe related to the heterogeneous structures observed at low Reynolds numbers (Van Loosdrecht et al., 2002). It could explain the sharp apparent permeability decrease at the early stage of the biofilter clogging in our case and the observed deviation of the Kozeny law.

Finally let us not forget some authors who invoke a phenomenon of junction clogging (Kapellos et al., 2007). In that case, depending on the structure built at the pore scale, the simple fact that some pores can be disconnected from the flow would cause a far more drastic permeability decrease than that caused by mere pore clogging. Indeed, when a junction clogs all the connected pores would become inaccessible to the flow, even if they contain no biofilm at all. In our case, we guess however that this phenomenon is not the main explanation for the sharp decrease that we observe in our experiments: we expect that junction clogging is more pronounced in the case when the size distribution of the grain in the porous media is quite large allowing larger heterogeneities in the pore size.

On the theoretical point of view, many works have been made on the derivation of permeability models accounting for the bioclogging of porous media. These models are briefly recalled in Appendix A and are presented in Fig. 12(b).

Most of the theoretical models (Clement et al., 1996; Thullner et al., 2002, 2004) are not able to represent our experimental data. This is partly explained by the biofilm representation in these models (uniform biofilm on the pore walls, bacterial colonies, 

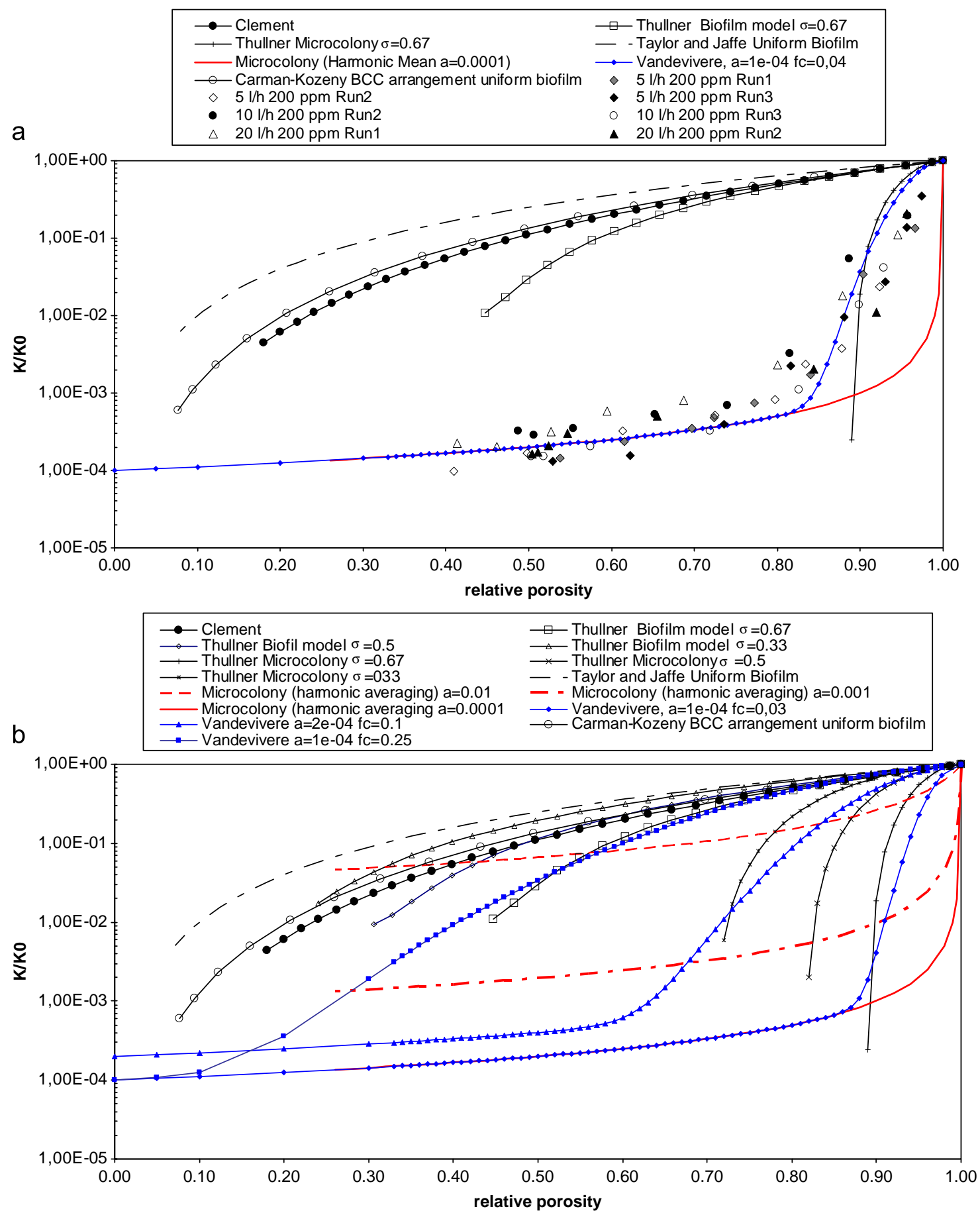

Fig. 12. (a) Experimental relationship between the relative permeability and the relative permeability. (b) Behaviour of different theoretical models of permeability accounting for bioclogging.

pore throat plugging) which prevent a universal representation of the complexity of the actual biofilm structure and distribution, which depend on the operating conditions and biomass physiology. Furthermore most of the assumptions behind these models consider that the Kozeny law is still valid for the clogged system. In particular, Clement et al. (1996) model exactly gives the same behaviour as the Kozeny law. It is likely that all these models do not properly represent the effect of the EPS on the permeability reduction as they are mainly based on geometrical considerations regarding the pore occupation by the biomass. Vandevivere's (1995) model seems to better approximate the experimental data. This model is also based on a given distribution of biomass within the pore but it allows a sudden transition between a "continuous biofilm" clogging mode and "pore throat plugged" mode. This transition is clearly seen on the fitted curve around $\phi / \phi_{0}=0.8$. For relatively high porosity reduction, the permeability seems to reach a "saturation" value. This saturation corresponds either to the biofilm permeability $K_{\text {min }}$ (the system is completely clogged and the water is actually flowing through the biofilm matrix itself) either to an effective permeability taking into account the existence of free spaces for the fluid inside the porous media: in this case the shear stress increases along with the 


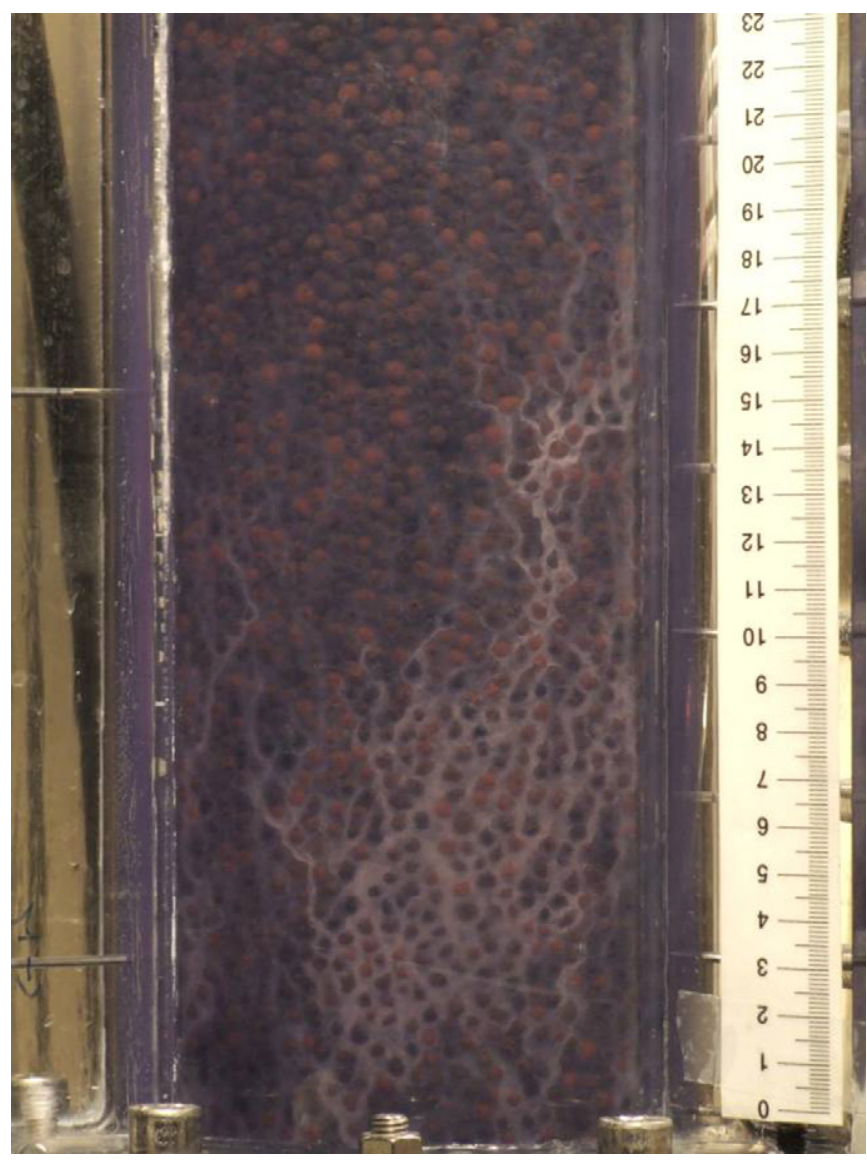

Fig. 13. Preferential path at the column wall.

permeability reduction and the detached mass of biofilm keep the pores open (Taylor and Jaffé, 1990). There would exist a steady state corresponding to a critical shear stress for which the net rate of biomass removal would balance the net biofilm growth. Another phenomenon, which is not incompatible with the open pore model, is the existence of channelling inside the biomass plugs. These channels were observed by Stewart and Fogler (2001) on their micro model and by the present authors by visual inspection of the column. Furthermore, the maximum permeability reduction observed in our experiments corresponds to a minimum macroporosity $\phi$ equal to 0.15 (curve not shown here). Fig. 13 was taken on our experiments: it clearly shows that channels do exist at the column wall, but this may be not representative of the phenomena occurring in the column core. This leads us to support the channelling effect and the open pore theory although more investigations are needed to quantify the relative importance of each individual process depending on the operating conditions.

As among all the existing permeability models, Vandevivere's (1995) model seems the most appropriate. This model can be used to derive a correlation which account for the bioclogging in the case of our experiment. This model writes (see Appendix A)

$\frac{K(\phi)}{K_{0}}=F\left(f_{x}\right)\left(1-f_{x}\right)^{2}+\left[1-F\left(f_{x}\right)\right]\left(\frac{a}{a+f_{x}-a f_{x}}\right)$

$F\left(f_{x}\right)=\exp \left(-0.5\left(f_{x} / f_{c}\right)^{2}\right)$

With, $\alpha=K_{\min } / K_{0}, f_{x}=1-\phi / \phi_{0} . f_{c}$ is a parameter to adjust. In our case, we get $\alpha=1 \mathrm{e}-04$ and $f_{c}$ was adjusted to 0.03 .

That result is important to supplement some models such as the models of Kildsgaard and Engesgaard (2001) and of Brovelli et al. (2009) which directly use a constitutive law between permeability and porosity to perform the coupling between the momentum equation and biomass growth. Kildsgaard and Engesgaard (2001) used Clement et al. (1996) formulation, but they calibrated their model only on the total pressure loss. It is then difficult to assess if another formulation of the pressure loss would have been better. However, Brovelli et al. (2009) tested different pressure loss formulation in their model which is mostly based on the same equations as Kildsgaard and Engesgaard (2001). Those formulations were the model of Clement et al. (1996) and the two models (microcolony and biofilm) of Thullner et al. (2004). They did not test Vandevivere (1995) formulation and that may explain some discrepancy between their simulations and the experimental data they used to test their model (the $2 \mathrm{D}$ dispersion of a passive tracer)

We also used the results of the simulation of Kapellos et al. (2007) (given in term of permeability evolution with time and porosity evolution with time) to compute the law $K(\phi)$ from their data. As it can be seen in Fig. 14, the numerical simulations of Kapellos et al. (2007) and our experimental data behave quite differently although the flow regime in both case corresponds almost to the same range of Reynolds number (Kapellos et al., 2007 simulation conditions: initial porosity $=0.56$, superficial velocity $=2 \mathrm{e}-03 \mathrm{~m} / \mathrm{s}$, grain diameter $=0.5 \mathrm{~mm}$, which gives an initial pore Reynolds number $R e_{p}=2.27$ ). It is difficult to decide which theoretical model is better to account for Kapellos et al. (2007) numerical data as most of them behave similarly at low porosity reduction. However, the microcolony model of Thullner et al. (2004) seems to better fit the simulated data. This seems coherent as Kapellos et al. (2007) only simulated the early stage of the porous media clogging and in their results, colonies around beads are clearly seen. As Kapellos et al. (2007) model includes the computation of the fluid flow inside the biofilm, ultimately, his simulation should exhibit a saturation when the porous media is completely plugged and the Vandevivere (1995) model with the appropriate parameters, should be able to capture this feature. Unfortunately, no simulations on the long term are presented in Kapellos et al. (2007) paper. Finally, the difference between Kapellos et al. (2007) and the present data at the early stage of the colonisation may be due to some feature of the biofilm structure which are present in our experiment but are not captured by the numerical model or not present in the conditions of the simulation : the simulation for instance does not show a very heterogeneous surface shape for the biofilm. These are again conjectures as we are not yet able to have an idea of the biofilm microstructure in our experiments.

As a conclusion of this paragraph, even if the Vandevivere (1995) model was able to recover our data behaviour, previous works show all the complexity in the biofilm structures and behaviour. This imply that a parameterization with only one parameter (namely the relative porosity) maybe not sufficient to account for all this complexity. Although existing formulations are useful and were somewhat used with some success in 1D models (Kildsgaard and Engesgaard, 2001 or Brovelli et al., 2009), we are far from having a general closure law for the permeabilitybiomass content relationship and as for the link between porosity and biomass content, this task requires work at a lower scale (pore and biofilm scale).

\subsection{Discussion}

Keeping in mind the trends presented in Section 2, the biomass and porosity profiles (Fig. 9), already used in a previous paragraph, are now analysed along with the oxygen profiles (Fig. 10) at the steady states. 


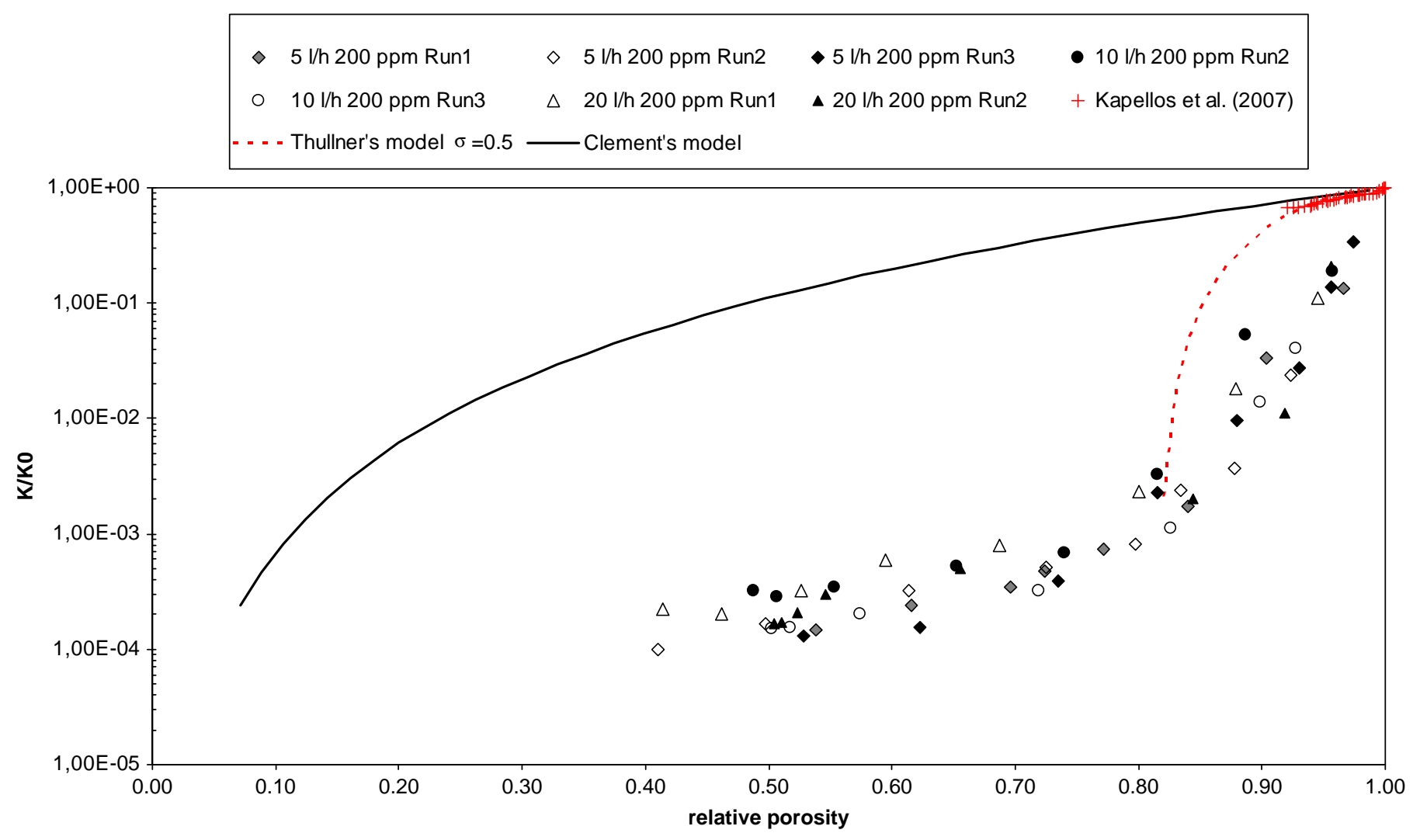

Fig. 14. Comparison of the numerical data of Kapellos et al. (2007) and our experimental data.

Data from Figs. 9 and 10 are discussed in the frame of a simple steady state section-averaged 1D model. Under the hypothesis of a local biomass growth, and biofilm detachment balanced by the net growth, simplified mass balances for the biomass (Eq. (9)) and oxygen (Eq. (10)) write

$\frac{\partial c_{x}}{\partial t}=r_{x} c_{x}-k_{\mathrm{det}} c_{x}$

$\phi \frac{\partial c_{o}}{\partial t}+\nabla\left(c_{o} \mathrm{v}\right)=-\phi_{0} \frac{r_{x}}{Y_{x 0}} c_{x}$

where $c_{x}$ is the mean biomass concentration (EPS+bacteria) in a cross section, $c_{o}$ the mean oxygen concentration at a given position and $v$ the superficial velocity. In Eq. (9), $r_{x}$ is the net biomass production rate and $k_{\text {det }}$ a function accounting for the detachment rate. In general, $k_{\text {det }}$ is related to many parameters accounting for the flow (shear stress $\tau$ ) and the biofilm structure (thickness $l_{b}$, biomass concentration $c_{x}$, biofilm density, mechanical resistance, etc.) (Kommedal and Blake, 2003). Concerning the growth rate, no assumption on the exact formulation of $r_{x}$ is introduced but we expect that function to depend on many parameters including physico-chemical parameters as well as possibly parameters linked to the hydrodynamic. In Eq. (10), $Y_{x 0}$ is an apparent yield coefficient for the oxygen and all dispersive terms were neglected. At the steady state, Eqs. (9) and (10) lead to

$r_{x}=k_{\operatorname{det}}(\tau, \ldots .$.

$v \nabla c_{0}=-\phi_{0} \frac{r_{x}}{Y_{x 0}} c_{x}$

Let us notice that in the equation above, $r_{x}, k_{\mathrm{det}}, Y_{x 0}$ are effective properties which integrate many processes linked to the cells and biofilm behaviour averaged at the biofilm scale then at the mesoscale.
From the experimental data presented in Figs. 9 and 10, the evolution of the ratio $r_{x} / Y_{x 0}$ was computed from Eq. (12). To perform this computation, profiles were fitted with smooth spline function. Only $z$ locations corresponding to strong gradients on the oxygen profiles were used in order to minimise the uncertainty. Fig. 15(a) shows the evolution of $r_{x} / Y_{x 0}$ along the column height for the 3 flow rates, while Fig. 15(b) presents the $z$-averaged of this quantity versus the flow rate.

It is clearly seen that the ratio $r_{x} / Y_{x 0}$ strongly evolves with the flow rate. The previous analysis cannot be applied to Eq. (11) because of the number of unknown parameters involved in the formulation of $k_{\text {det }}$. On the other hand, if the biomass balance must be fulfilled, Eq. (11) shows also that the net growth rate must depend on the hydrodynamic conditions.

Many simplified models which are directly derived at the macroscale and which do not rely on averaging processes or upscaling techniques use classical formulation for the kinetics, with constant parameters. For instance, the net growth rate $r_{x}$ is usually based on the Monod kinetic law or a related formulation. In our case, if we take one of the most complete law (namely the Haldane law in order to take into account possible bacteria inhibition by phenol), we get

$r_{x}=\mu_{\max } \frac{c_{s}(z)}{k_{s}+c_{s}(z)+c_{s}(z)^{2} / k_{i}} \frac{c_{0}(z)}{c_{0}(z)+k_{0}}-\mu_{\text {decay }}$

where $\mu_{\text {max }}, \mu_{\text {decay }}, k_{s}, k_{i}$ and $k_{o}$ are the maximum bacteria growth rate, the biomass mortality rate, the phenol saturation coefficient, the phenol inhibition coefficient and the oxygen saturation coefficient, respectively. This formulation does not explicitly involve hydrodynamic effect such as those due to the shear stress, or the flow conditions. In our case, given the phenol and the oxygen concentrations, given the observed phenol reduction in our operating conditions, and given the range of values found for these kinetics parameter in the literature (Iliuta and Larachi, 
a

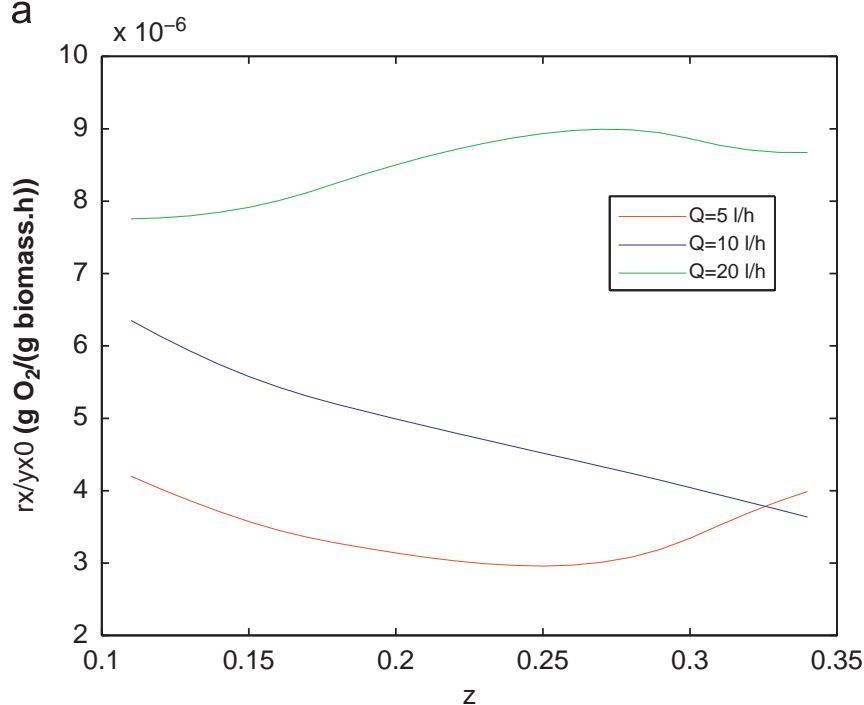

b

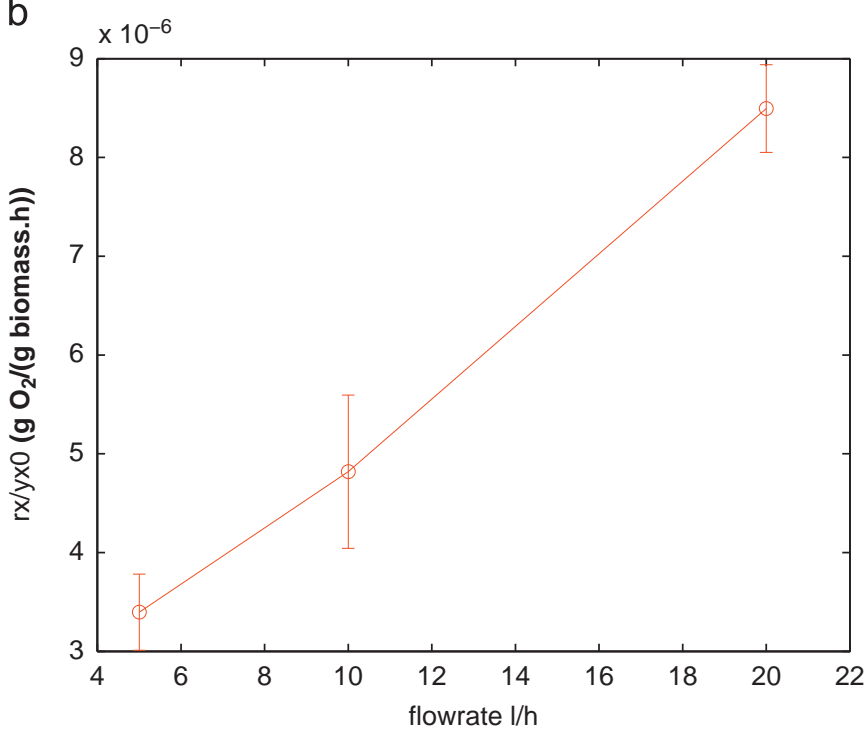

Fig. 15. (a) $r_{x} / Y_{x 0}=f(z)$ and (b) $r_{x} / Y_{x 0}=f(Q)$.

2005) using those values, this term appears to be nearly constant (around $0.35 \mathrm{~h}^{-1}$ ). The measured evolutions with the flow rate cannot be then explained through such a simple kinetic model which is strictly speaking valid at the local scale except if those parameters are at least flow dependent. This is not a surprise as $r_{x}$, $Y_{x 0}$, etc. are closure terms resulting on a succession of averaging on different scales.

Some authors (Zysset et al., 1994; Kildsgaards and Engesgaard, 2001; Brovelli et al., 2009) overcome such difficulties linked to the modelling of biofilm growth, and the introduction of closure term, by the use of a limiting $\eta$ function on the growth rate $r_{x}$. This limiting function accounts at the macroscopic scale for the change in the biofilm diffusive properties, surface exchange, substrate availability and the amount of active biomass as the biofilm grows: this change in these properties is directly linked to the change in the effective biofilm growth rate. We checked if, by introducing such a formulation, the experimental data could be explained from Eq. (11) and Eq. (12). The limiting function is often chosen as a function of the porous media actual biomass content and maximum biomass content that a pore can sustain. In the general case, $\eta$ can be written in term of biomass concentration (Kildsgaards and Engesgaard, 2001). Writing $\eta$ in term of porosity, we get

$\eta=\frac{c_{x, \max }-c_{x}}{c_{x, \max }}=\frac{\rho_{\max }\left(\omega-\rho_{x} / \rho_{\max }\right) \phi_{0}+\rho_{x} \phi}{\rho_{\max } \omega \phi_{0}}$

where $\omega$ is the maximum pore fraction occupied with biomass, $\rho_{\text {max }}$ and $\rho_{x}$ the biofilm density corresponding to the concentration $c_{x, \max }$ and $c_{x}$. As the biofilm density is constant in our experiments, the above equation can be simplified into

$\eta=\frac{(\omega-1) \phi_{0}+\phi}{\omega \phi_{0}}$

Eq. (14) or (15) states that the more the biofilm is thick, the more the effective growth rate decrease. Using the correction given by Eq. (15), the biomass and oxygen balance give

$r_{x} \frac{(\omega-1) \phi_{0}+\phi}{\omega \phi_{0}}=k_{\operatorname{det}}(\tau, \ldots .$.

$v \nabla c_{0}=-\phi_{0} \frac{r_{x}}{Y_{x 0}} \frac{(\omega-1) \phi_{0}+\phi}{\omega \phi_{0}} c_{x}$

It was checked if the use of such a limitation function allowed to explain the experimental data. For that purpose, the ratio $r_{x} / Y_{x 0}$ was computed following the same procedure as before. The value of $\omega$ was deduced from the experimental porosity profile at $z=0$.

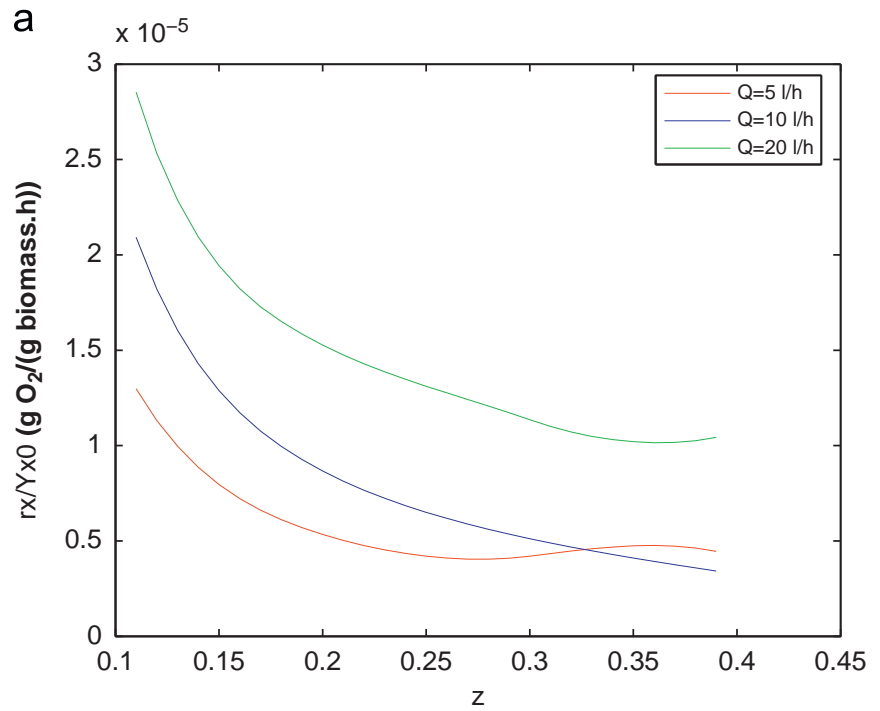

b

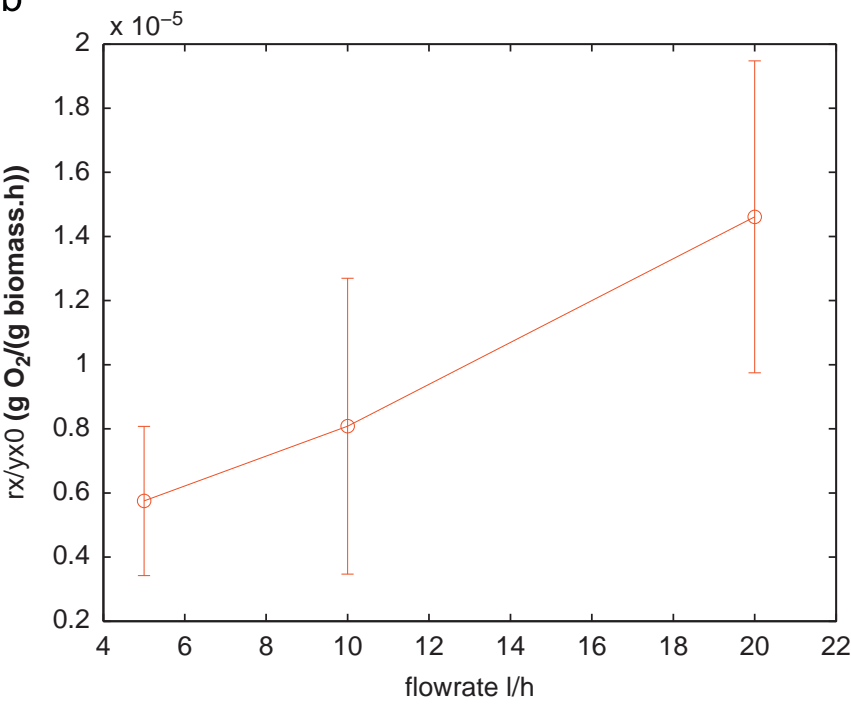

Fig. 16. Evolution of the corrected ratio $r x / Y_{x 0}$. 
The results are presented in Fig. 16. The ratio $r_{x} / Y_{x 0}$ is still dependent on the flow rate. Its dependency with the column depth is currently impossible to interpret: as already said, the number of unknown parameters involved in biomass balance equation (Eq. (16)) prevent an accurate analysis of the growth kinetic $r_{x}$ in regards of the detachment rate $k_{\text {det }}$. However, results shows clearly that, although convenient in the frame of a 1D dimensional model, the use of a limiting function along a Monodlike formulation for the growth kinetics would still result in flow dependent parameters for the different kinetic parameter. Indeed, given its very simple formulation, $\eta$ appears more as a convenient mathematical way to allow the system to reach a steady state than a function which can account for all the complex behaviour of the biofilm at the mesoscale. All the "hidden" processes, not well accounted for by this limiting function, are recovered in the flow dependence of the parameter in the Monod law. A resulting question is the validity of the assumption which postulate a priori that the term $r_{x}$ can be written from a formulation similar to the Monod-law, or if this term must be sought under a more general form (which is unknown). Let us stress that, in our experiments, given our very low Reynolds number values and narrow range of investigated Reynolds number, it is very probable that mass transfer toward the biofilm, the biofilm structure itself as well as the mass transfer within the biofilm do not change significantly. This conjecture is grounded on a comparison of our experimental data, with those of Garcia Lopez et al. (2003) for a biofilm grown on a membrane in laminar conditions. Indeed, for those conditions, Garcia Lopez et al. (2003) did not observed a significant mass transfer evolution for Reynolds number below 190. The wet biofilm volumetric density remained almost constant (around $30 \mathrm{~g} \mathrm{l}^{-1}$ as in our experiment) but a neat increase on the biomass content (biofilm thickness equal to 361, 418 and $495 \mu \mathrm{m}$, respectively) was observed. Our measurements and interpretations seem consistent with these results. Our observations cannot be explained by a variation of the term $Y_{x 0}$ but rather than an evolution of the term $r_{x}$ with the flow condition as stated by Eq. (9). At the current state a deeper analysis is not possible, as in Eq. (9) the closure law for $k_{\text {det }}$ includes too many unknown processes and parameters. Some conjectures can however be made from recent knowledge about biofilms found in literature. There are indeed many works in the literature which focus on the effect of the hydrodynamic on biofilm structure (Peyton, 1996; Van Loosdrecht et al., 2002). The most recent works deal explicitly on the change induced by the local hydrodynamic on the biofilm metabolism (Simoes et al., 2007, Liu and Tay, 2002). In particular, the shear stress would stimulate the production of EPS (Qi et al., 2008). This phenomenon could explain the $r_{x} / Y_{x 0}$ dependency with the column depth as well as with the flow rate observed in Figs. 15 and 16. However, on our experiment, without more information at the pore scale, this possible explanation is only a speculation at the present time.

\section{Conclusions and perspectives}

In this work, a laboratory scale bioreactor was presented as well as a database resulting of controlled experiments performed on that pilot. The consistency of the data was carefully checked and the results allowed the study of some features concerning the biofilm structure within the biofilter.

The raw data showed the existence of a steady state. In particular, the clogging front reached a stable position, which was interpreted as an equilibrium between growth and detachment, the detached fragment beings not recaptured downstream in the porous media. Some features concerning the bioreactor behaviour under different flow conditions were deduced from this steady state.

First, the independent measurement of porosity and biomass concentration (in term of total solid) showed a very simple relationship between these two quantities allowing the computation of the volumetric biofilm density. This value is not very sensitive to the operating conditions in the range of Reynolds number which were investigated. This result does not allow us however to discuss the evolution of the biofilm structure with the operating conditions and local flow conditions: parameters not measured here can be involved and the knowledge of more refined data (among them the evolution of the EPS/bacteria cells ratio, and the biomass distribution within a section) are required to discuss this point. In particular, the linear relationship found in the present work is probably not a general behaviour and remains specific to our operating conditions.

Second, the relationship between the column permeability and porous media porosity was then checked and the experimental results were confronted to existing theoretical models and numerical simulations. The reduction of permeability is very sharp as soon as the biofilm appears, at the early stage of beads colonisation. From the current knowledge about biofilms behaviour in porous media, this sharp decrease may involve complex behaviours related with the existence of biopolymers as well as the existence of a complex microstructure at the pore scale. This explains that a Kozeny like formulation fails to predict the permeability reduction with the biomass content. The Vandevivere (1995) model is the most appropriate in the case of our experiment. As for the volumetric density, the permeabilityporosity relationship is not sensitive to the operating conditions in our case but the Reynolds number range investigated is quite low leading maybe to a heterogeneous biofilm in all cases. The sensitivity of this law to the biofilm structure at the pore scale as well as the biomass distribution at the pore scale is then still an open question and requires more data on a larger range of operating conditions. In particular, it may be not sufficient to relate the permeability to a single global parameter such as the macroporosity. Although not general, our results were compared with an advanced recent numerical model (for the same range of pore Reynolds number). This later model was based on a refined description of the biofilm at various scales but still the modelled interaction fluid/biofilm do not capture yet the sharp permeability decrease observed on our experimental data. The predicted permeability reduction is still lower than those observed on the experimentation. The simulations show the existence of colonies then patches which grows around the beads and within the pore. The simulated data can be then approached by theoretical models involving such microcolonies. The observed difference compared to our experimentations lie then maybe in a microstructure which is not yet accurately captured by these refined numerical models.

The experimental permeability reduction reaches a saturation value which can be interpreted following several concept depending on whether the pores are considered to be completely filled (close pore theory) or if some pores remain available to the flow due to an equilibrium between the biofilm growth and the detachment rate (open pore theory). From the coupled measurements of permeability and porosity reduction, our experiments would favour the open pore theory (with possibly, the existence of channelling inside the plug part of the column, as inferred by Stewart and Fogler (2001) in their micro-scale experiments).

Finally, the steady state profiles were analysed in the frame of a simple 1D model. It was shown that classical kinetics law accounting for cells growth (Monod, Haldane laws) or biofilm growth rate (through the use of a limiting function) could not explain our experimental data, if the biomass growth rate is not flow rate (or shear) dependent. The failure of these classical laws 
to explain our measurements lies in the fact that, often, they mix concepts which are valid at the local scale with heuristic concepts (limitation function for instance) accounting for the rigorous averaging procedure which should be performed to properly derive a macroscale model. All the closure laws required in the model are effective properties, whose structures cannot be postulated a priori as they result of many processes occurring at the biofilm and cell scale, averaged at the macroscale. Those processes depend on many hydrodynamical and biological factors.

In our case, the increase of the total amount of biomass with the flow rate could be interpreted as the bacteria biological response to the local hydrodynamic. Among the involved process in this biological response, a possible answer consists in an increase in the polymer production as the flow rate increases and/or metabolic changes within the biofilm as the shear stress increases. This conclusion is purely conjectural but consistent with recent results about biofilm behaviour (EPS production: Qi et al., 2008, growth kinetics: Tsai, 2005, biofilm phenotype: Simoes et al., 2007). To get realistic models, describing separately the EPS and the bacterial cells seems necessary, as well as including phenomena linked to the coupling between the cells metabolism and the flow (growth rate, EPS production, gene activation, etc.). Some models begin to include separated descriptions of the EPS and bacterial cells, but the kinetics are still independent on the flow. To our knowledge, the only model to include such effects is Kapellos et al. (2007) model (through pressure inhibition growth effects and cells-to-cells signalling molecules productions). Even in that case, as it has been seen, some features such as the permeability reduction prediction are not in accordance with our observed data. In the current states, our data cannot be fully explained without a deep knowledge of many coupled mechanisms occurring at various scales, from the cell/biofilm scale to the reactor scale.

\section{Appendix A. Permeability reduction due to biomass growth in porous media. Existing theoretical model}

A first set of models is based on the concept of uniform biofilm and gives a power law relationship between the relative permeability and relative porosity

$\frac{K}{K_{0}}=\left(1-f_{x}\right)^{n}=\left(\frac{\phi}{\phi_{0}}\right)^{n}$

For an idealised porous medium made of identical cylindrical tubes, one gets $n=2$ from the Hagen-Poiseuille law. Using this concept, Taylor and Jaffé (1990) followed the same approach as Ives and Pienvichitr (1965) for colloids trapped in a porous medium: they considered a porous medium made of spheres of uniform size and various beads arrangements. The biofilm is considered to be uniformly distributed around the beads. Geometrical consideration about the colonized beads diameter and packing porosity were used to supplement the Carman-Kozeny law and to follow its evolution with the biomass content (in particular the biofilm thickness). The relative permeability reduction with the relative porosity was found poorly sensitive to the beads arrangement, and at the first order, provided the porosity is not too low, their model can be approximated by a power law (as in Eq. (A1)) with $n \sim 3 / 2$. In another model, Clement et al. (1996) did not assume a specific pattern for the biomass distribution. From an approach based on the macroscopic interpretation of the "cut and random rejoin" model presented also in Taylor and Jaffé (1990), they derived an expression similar to the uniform biofilm concept with $n=19 / 6$.
Thullner et al. (2002) followed the same approach using a network of interconnected capillaries of random diameter. The pressure drop in each capillary was computed using the Poiseuille law and solving convection-diffusion equation for the increase of biomass concentration. The capillaries geometry was modified accordingly assuming a uniform growth on the capillaries wall. The global pressure drop (against the biomass content or porosity) can be then computed at each time step. The result writes

$\frac{K(\phi)}{K_{0}}=\left[\left(\frac{\phi / \phi_{0}-\alpha_{0}}{1-\alpha_{0}}\right)^{b}+\frac{K_{\min }}{K_{0}}\right] \frac{1}{1+K_{\min } / K_{0}}$

$\phi_{0}, K_{\min }$ and $\alpha_{0}$ are adjustable parameters, which depend on the pore size distribution standard deviation.

Other models are based on the "microcolony" concept. Following this concept, the biofilm is not uniformly distributed around the media but forms a plug in the capillaries or pore throat. A simple version of this approach considers a set of tubes divided between a clean part (permeability $K_{0}$ ) and a plugged part (permeability $K_{\min }$ ). The overall permeability $K$ depends on the space filled by the plug (hence on the porosity $\phi$ ). This permeability is expressed as the harmonic mean between the clean and plugged part, which yields

$\frac{K(\phi)}{K_{0}}=\frac{a}{a+f_{x}-a f_{x}}$

with $a=K_{\min } / K_{0} . f_{x}$ is the biomass fraction (relative to the pore volume) defined as: $f_{x}=1-\phi / \phi_{0}$.

Thullner et al. (2002) considered also a scenario based on the concept of "microcolony". Using their network approach, the clogging process was simulated by randomly selecting a volume of biomass and removing the number of pores necessary to reach this volume. Smaller pores were removed preferentially. For each realisation, the flow and permeability change were then computed. The analysis of simulated data led to

$\frac{K(\phi)}{K_{0}}=\alpha_{t}\left(\frac{\phi / \phi_{0}-\alpha_{0}}{1-\alpha_{0}}\right)^{3}+\left(1-\alpha_{t}\right)\left(\frac{\phi / \phi_{0}-\alpha_{0}}{1-\alpha_{0}}\right)^{2}$

$\alpha_{t}$ and $\alpha_{0}$ are adjustable parameters depending on the pore size distribution standard deviation.

Finally, Vandevivere (1995) stated that a permeability model combining the two approaches (uniform biofilm and microcolony) would better fit the data. His model is based on the assumption that at low bacterial content, cells multiply under the form of a biofilm. As the shear stress increases (because of the pore reduction) detached part of the biofilm can create plugs downstream. The model itself is derived considering a set of capillaries. At low bacterial density, most of the capillaries are treated considering the biofilm model. As the biomass fraction $f_{x}$ increases, a fraction of the tube is described from the plug model. If $F\left(f_{x}\right)$ represents the fraction of pores that do not yet present a plug for a given biomass fraction $f_{x}$, the relative permeability is computed from the weighted average of the biofilm model (Eq. (A1)) and plug model (Eq. (A3)). This yields

$\frac{K(\phi)}{K_{0}}=F\left(f_{x}\right)\left(1-f_{x}\right)^{2}+\left[1-F\left(f_{x}\right)\right]\left(\frac{a}{a+f_{x}-a f_{x}}\right)$

with, $a=K_{\min } / K_{0}$ and $f_{x}=1-\phi / \phi_{0}$.

$F\left(f_{x}\right)$ is a decreasing function theoretically equal to 1 when $f_{x}=0$ (no plugs at low biomass fraction) and equal to 0 when $f_{x}=1$ (all pores are plugged). Vandevivere choose a sigmoïdal function corresponding to the right half of a normal distribution

$F\left(f_{x}\right)=\exp \left(-0.5\left(f_{x} / f_{c}\right)^{2}\right)$

This function physically corresponds to a gradual change of the permeability reduction as the biofilm thickens until a critical biomass fraction $f_{c}$ is reached. Then the model switches from the 
biofilm model to the plug model and the critical value corresponds, in the authors view, to a biofilm instability. Such behaviour at a critical biomass content was also suggested by Characklis et al. (1987).

\section{References}

Anthony, P., Geindreau, C., Séchet, Ph., Martins, J., 2005. Biofilm growth in porous media: derivation of a macroscopic model from the physics at the pore scale via homogeneization. In: Kennes, Christian, Veiga, Maria C. (Eds.), Proceedings of the International Congress Biotechniques for Air Pollution Control, October 5-7, La Coruna, Spain. A Coruna. Universida da Coruna, Servizo de Publications Publ., ISBN: 84-9749-163-7, pp. 171-179.

Bertani, G., 1951. Studies on lysogenesis I: the mode of phage liberation by lysogenic Escherichia coli. Journal of Bacteriology 62 (3), 293-300.

Bielefeldt, A., McEachern, C., Illangasekare, T., 2002. Hydrodynamic changes in sand due to biogrowth on naphthalene and decane. Journal of Environmental Engineering 128 (1), 51-59.

Bordas, M.L., Cartellier, A., Sechet, P., Boyer, C., 2006. Bubbly flow through fixed beds: microscale experiment in the dilute regime and modeling. AIChE Journal $52,3722-3743$

Brovelli, A., Malaguerra, F., Barry, D.A., 2009. Bioclogging in porous media: model development and sensitivity to initial conditions. Environmental Modelling and Software 24, 611-626.

Characklis, W.G., Cunningham, A.B., Escher, A., Crawford, D., 1987. Biofilms in porous media. In: Cullimore, D.R. (Ed.), Proceedings of the International Symposium on Biofouled Aquifers: Prevention and Restoration. American Water Resources Association Technical Publication Series. American Water Resources Association, Bethesda, MD, pp. 57-78.

Chen-Charpentier, B., 1999. Numerical Simulation of biofilm growth in porous media. Journal of Computational and Applied Mathematics 103, 55-66.

Clement, T.P., Hooker, B.S., Skeen, R.S., 1996. Macroscopic models for predicting changes in saturated porous media properties caused by microbial growth. Ground Water 34, 934-942.

Cunningham, A., Characklis, W.G., Abedeen, F., Crawford, D., 1991. Influence of biofilm accumulation on porous media hydrodynamics. Environmental Science \& Technology 25, 1305-1311.

Davies, D.G., Parsek, M.R., Pearson, J.P., Iglewski, B.H., Costerton, J.W., Greenberg, E.P., 1998. The involvement of cell-to-cell signals in the development of a bacterial biofilm. Science 280, 295-298.

Deront, M., Samb, F.M., Adler, N., Péringer, P., 1998. Volumetric oxygen mass transfer coefficient in an upflow cocurrent packed-bed bioreactor. Chemical Engineering Science 53, 1321-1330.

Di Iaconi, C., Ramadori, R., Lopez, A., Passino, R., 2006. Influence of hydrodynamic shear forces on properties of granular biomass in a sequencing batch biofilter reactor. Biochemical Engineering Journal 30, 152-157.

Garcia Lopez, L.A., Veiga, M.C., Nogueira, R., Aparicio, A., Melo, L.F., 2003. A technique using a membrane flow cell to determine average mass transfer coefficients and tortuosity factors in biofilms. Water Science and Technology 47 (5), 61-67.

Golfier, F., Wood, B.D., Orgogozo, L., Quintard, M., Bues, M., 2009. Biofilms in porous media: development of macroscopic transport equations via volume averaging with closure for local mass equilibrium conditions. Advances in Water Resource 32 (3), 463-485.

Hill, G.A., Robinson, C.W., 1975. Substrate inhibition kinetics: phenol degradation by Pseudomonas putida. Biotechnology and Bioengineering 17, 1599-1615.

Iliuta, I., Larachi, F., 2005. Modelling simultaneous biological clogging and physical plugging in trickle-bed bioreactors for wastewater treatment. Chemical Engineering Science 60, 1477-1489.

Ives, K.J., Pienvichitr, V., 1965. Kinetics of the filtration of dilute suspensions. Chemical Engineering Science 20, 965-973.

Kapellos, G.E., Alexiou, T.S., Payatakes, A.C., 2007. A multiscale theoretical model for diffusive mass transfer in cellular biological media. Mathematical Biosciences 210, 177-237.

Karrabi, M., 2009. Coupling between hydrodynamic and biofilm in porous media: application to a biofilter. Ph.D. Thesis, LEGI, University of Grenoble, France.
Karrabi, M., Sechet, P., Morra, Ch., Florensa, I., Geindreau, Ch., Martins, J., Cartellier A., 2006. Experimental investigation of biofilm growth and hydrodynamic biomass interaction in a granular bioreactor. In: Proceedings of the Sixth Chisa Congress, Pranha, Czech Republic.

Kildsgaard, J., Engesgaard, P., 2001. Numerical analysis of biological clogging in twodimensional sand box experiments. Journal of Contaminant Hydrology 50 261-285

Liu, Y., Tay, J.H., 2002. The essential role of hydrodynamic shear force in the formation of biofilm and granular sludge. Water Research 36, 1653-1665.

Menoret, C., 2001. Traitement d'effluents concentrés par cultures fixées sur gravier ou pouzzolane, Ph.D. Thesis, University of Montpellier II (in French).

Ortiz-Arroyo, A., Larachi, F., Iliuta, I., 2003. Method for inferring contact angle and for correlating static liquid hold-up in packed beds. Chemical Engineering Science 58, 2835-2855.

Peyton, B.M., 1996. Effects of shear stress and substrate loading rate on pseudomonas aeruginosa biofilm thickness and density. Water Research 30, 29-36.

Picioreanu, C., 1999. Multidimensional modelling of biofilm structure, Ph.D. Thesis, Delft University of Technology, The Netherlands.

Picioreanu, C., van Loosdrecht, M.C., Heijnen, J.J., 2000. Effect of diffusive and convective substrate transport on biofilm structure formation: a two-dimensional modeling study. Biotechnology and Bioengineering 69, 504-515.

Qi, P., Wang, W., Qi, Z., 2008. Effect of Shear Stress on Biofilm Morphological Characteristics and the Secretion of Extracellular Polymeric Substances, 978-14244-1748-3/08/2008 IEEE.

Shaw, J.C., Bramhill, B., Wardlaw, N.C., Costerton, J.W., 1985. Bacterial fouling in a model core system. Applied and Environmental Microbiology 49, 693-701.

Siegrist, H., Gujer, W., 1985. Mass transfer mechanisms in a heterotrophic biofilm. Water Research 19 (11), 1369-1378.

Simoes, M., Peireira, M.O., Sillankorva, S., Azeredo, J., Vieira, M.J., 2007. The effect of hydrodynamic conditions on the phenotype of Pseudomonas fluorescens biofilms. Biofouling 23 (3/4), 249-258.

Stewart, T.L., Fogler, H.S., 2001. Biomass plug development and propagation in porous media. Biotechnology and Bioengineering 72 (3), 353-363.

Taylor, S.W., Jaffé, P.R., 1990. Biofilm growth and the related changes in the physical properties of a porous medium. 1. Experimental investigations. Water Resource Research 26, 2153-2159.

Thullner, M., Mauclaire, L., Schroth, M.H., Kinzelbach, W., Zeyer, J., 2002. Interaction between water flow and spatial distribution of microbial growth in a twodimensional flow field in saturated porous media. Contaminant Hydrology 58 169-189.

Thullner, M., Schroth, M.H., Zeyer, J., Kinzelbach, W., 2004. Modeling of a microbial growth experiment with bioclogging in a two-dimensional saturated porous media flow field. Contaminant Hydrology 70, 37-62.

Tsai, Y.-P., 2005. Impact of flow velocity on the dynamic behaviour of biofilm bacteria. Biofouling 21 (5/6), 267-277.

Van Loosdrecht, M.C.M., Heijnen, J.J., Eberl, H., Kreft, J., Picioreanu, C., 2002. Mathematical modelling of biofilm structures. Antonie van Leeuwenhoek 81, 245-256.

Vandevivere, P., Baveye, P., 1992. Saturated hydraulic conductivity reduction caused by aerobic bacteria in sand columns. Soil Science Society of America $56,1-13$

Vandevivere, P., Baveye, P., de Lozada, D.S., DeLeo, P., 1995. Microbial clogging of saturated soils and aquifer materials: evaluation of mathematical models. Water Resource Research 31, 2173-2180.

Vandevivere, P., 1995. Bacterial clogging of porous media: a new modelling approach. Biofouling 8, 281-291.

Venugopalan, V.P., Kuehn, M., Hausner, M., Springael, D., Wilderer, P.A., Wuertz, S. 2005. Architecture of a nascent Sphingomonas sp. biofilm under varied hydrodynamic conditions. Applied and Environmental Microbiology 71 (5), 2677-2686.

Wanner, O., Eberl, H.J., Morgenroth, E., Noguera, D.R., Picioreanu, C., Rittmann, B.E., Van Loosdrecht, M.C.M., 2004. Mathematical Modeling of Biofilms, IWA Biofilm Specialists Conference in Las Vegas, Nevada, USA

Wood, B.D., Ford, R.M., 2007. Biological processes in porous media: from the pore scale to the field. Advances in Water Resource 30 (6-7), 1387-1391.

Zysset, A., Stauffer, F., Dracos, T., 1994. Modeling of reactive groundwater transport governed by biodegradation. Water Resour. Res. 30, 2423-2434. 